\title{
Hamilton Jacobi equations on metric spaces and transport entropy inequalities
}

\author{
Nathael Gozlan, Cyril Roberto and Paul-Marie Samson
}

\begin{abstract}
We prove a Hopf-Lax-Oleinik formula for the solutions of some Hamilton-Jacobi equations on a general metric space. As a first consequence, we show in full generality that the log-Sobolev inequality is equivalent to a hypercontractivity property of the Hamilton-Jacobi semi-group. As a second consequence, we prove that Talagrand's transport-entropy inequalities in metric space are characterized in terms of log-Sobolev inequalities restricted to the class of $c$-convex functions.
\end{abstract}

\section{Introduction}

Let $L: \mathbb{R}^{m} \rightarrow \mathbb{R}$ be a convex function with superlinear growth, in the sense that $L(h) /\|h\| \rightarrow \infty$, when $\|h\| \rightarrow \infty$, where $\|\cdot\|$ is any norm on $\mathbb{R}^{m}$. It is well known that if $f$ is some Lipschitz function on $\mathbb{R}^{m}$, the function $Q_{t} f$ defined by

$$
Q_{t} f(x)=\inf _{y \in \mathbb{R}^{m}}\{f(y)+t L((x-y) / t)\}, \quad t \geq 0, x \in \mathbb{R}^{m},
$$

is a solution, in different weak senses, of the following Hamilton-Jacobi equation,

$$
\partial_{t} u(t, x)=-L^{*}\left(\partial_{x} u(t, x)\right)
$$

with initial condition $u(0, x)=f(x)$, where $L^{*}(v)=\sup _{u \in \mathbb{R}^{m}}\{u \cdot v-L(u)\}$ is the Fenchel-Legendre transform of $L$ (see for instance [7]). It can be shown, for example, that the function $(t, x) \mapsto Q_{t} f(x)$ is almost everywhere differentiable in $(0, \infty) \times \mathbb{R}^{m}$ and that (1.2) is satisfied at every point of differentiability (see, e.g., Chapter 3 of [7]). Formula (1.1) is usually referred to as the Hopf-Lax-Oleinik formula for Hamilton-Jacobi equations.

The objectives of this paper are two:

(i) to generalize the Hopf-Lax-Oleinik (HLO) formula to a class of HamiltonJacobi equations in a metric space framework;

Mathematics Subject Classification (2010): Primary 60E15; Secondary 47H20.

Keywords: Transport inequalities, Hamilton-Jacobi equations, logarithmic Sobolev inequalities, metric spaces. 
(ii) to use this HLO formula to establish different connections between logarithmic Sobolev type inequalities and transport-entropy inequalities.

\subsection{General framework}

In this section we give the general setting of this article.

1.1.1. Assumptions on the space. Throughout the paper, $(X, d)$ will be a complete and separable metric space in which closed balls are compact. This last assumption could be removed at the expense of additional standard technicalities. We will sometimes assume that $(X, d)$ is a geodesic space, meaning that for every two points $x, y \in X$ there is at least one curve $\left(\gamma_{t}\right)_{t \in[0,1]}$ with $\gamma_{0}=x$ and $\gamma_{1}=y$, and such that $d\left(\gamma_{s}, \gamma_{t}\right)=|t-s| d(x, y)$ for all $s, t \in[0,1]$. Such a curve is called a geodesic between $x$ and $y$.

1.1.2. The sup and inf convolution "semigroups". Throughout the paper, $\alpha: \mathbb{R}^{+} \rightarrow \mathbb{R}^{+}$will be an increasing convex function of class $\mathcal{C}^{1}$ such that $\alpha(0)=0$. If $f: X \rightarrow \mathbb{R}$ is a bounded function, we define, for all $t>0$, the functions $P_{t} f$ and $Q_{t} f$ by

$$
P_{t} f(x)=\sup _{y \in X}\left\{f(y)-t \alpha\left(\frac{d(x, y)}{t}\right)\right\}, \quad \forall x \in X,
$$

and

$$
Q_{t} f(x)=\inf _{y \in X}\left\{f(y)+t \alpha\left(\frac{d(x, y)}{t}\right)\right\}, \quad \forall x \in X
$$

The operators $P_{t}$ and $Q_{t}$ are connected by the following simple relation:

$$
Q_{t} f=-P_{t}(-f)
$$

When the space $(X, d)$ is geodesic, the families of operators $\left\{Q_{t}\right\}_{t>0}$ and $\left\{P_{t}\right\}_{t>0}$ form nonlinear semigroups acting on bounded functions:

$$
Q_{t+s} f=Q_{t}\left(Q_{s} f\right) \quad \text { and } \quad P_{t+s} f=P_{t}\left(P_{s} f\right), \quad \forall t, s>0,
$$

for all bounded function $f: X \rightarrow \mathbb{R}$. When $(X, d)$ is not geodesic, only half of this property is preserved:

$$
Q_{t+s} f \leq Q_{t}\left(Q_{s} f\right) \quad \text { and } \quad P_{t+s} f \geq P_{t}\left(P_{s} f\right), \quad \forall t, s>0 .
$$

Now we present our main results.

\subsection{A Hopf-Lax-Oleinik formula on a metric space}

Our objective is to show that the Hamilton-Jacobi equation (1.2) is still satisfied by $Q_{t} f$ in the metric space framework introduced above. To that purpose we first need to give a meaning to the state space partial derivative $\partial_{x}$ in this context. 
We will adopt the classical measurements $\left|\nabla^{+} f\right|(x)$ and $\left|\nabla^{-} f\right|(x)$ of the local slope of a function $f: X \rightarrow \mathbb{R}$ around $x \in X$ defined by

$$
\left|\nabla^{+} f\right|(x)=\limsup _{y \rightarrow x} \frac{[f(y)-f(x)]_{+}}{d(x, y)}, \quad\left|\nabla^{-} f\right|(x)=\limsup _{y \rightarrow x} \frac{[f(y)-f(x)]_{-}}{d(x, y)}
$$

(by convention, $\left|\nabla^{ \pm} f\right|(x)=0$ if $x$ is an isolated point in $X$ ).

If $f$ is locally Lipschitz, then $\left|\nabla^{ \pm} f\right|(x)$ are finite for every $x \in X$. Moreover, if $f$ is Lipschitz continuous with Lipschitz constant $\operatorname{Lip}(f)$, then $\left|\nabla^{ \pm} f\right|(x) \leq \operatorname{Lip}(f)$ for all $x \in X$. Finally, when $X$ is a Riemannian manifold and $f$ is differentiable at $x$, it is not difficult to check that $\left|\nabla^{ \pm} f\right|(x)$ is equal to the norm of the vector $\nabla f(x) \in T_{x} X$ (the tangent space at $x$ ).

One of our main results is the following theorem.

Theorem 1.1. If $f: X \rightarrow \mathbb{R}$ is an upper semicontinuous function bounded from above, then there hold the following Hamilton-Jacobi differential inequalities:

$$
\frac{d}{d t_{+}} P_{t} f(x) \geq \alpha^{*}\left(\left|\nabla^{+} P_{t} f\right|(x)\right) \quad \forall t>0, \quad \forall x \in X
$$

and

$$
\frac{d}{d t_{-}} P_{t} f(x) \geq \alpha^{*}\left(\left|\nabla^{-} P_{t} f\right|(x)\right) \quad \forall t>0, \quad \forall x \in X
$$

where $\alpha^{*}(u)=\sup _{h \geq 0}\{h u-\alpha(h)\}, u \geq 0$, and where $d / d t_{+}$and $d / d t_{-}$denote respectively the right and left time derivatives.

Moreover, when the space $(X, d)$ is geodesic, there holds

$$
\frac{d}{d t_{+}} P_{t} f(x)=\alpha^{*}\left(\left|\nabla^{+} P_{t} f\right|(x)\right) \quad \forall t>0, \quad \forall x \in X .
$$

The interesting feature of Theorem 1.1 is that there is no measure theory in its formulation: the conclusion holds for all $t>0$ and all $x \in X$. Theorem 1.1 extends previous results by Lott and Villani ([14], [20]), where (1.7) was obtained on compact measured geodesic spaces $(X, d, \mu)$ provided the measure $\mu$ satisfies some additional assumptions. More precisely, it is proved in [14] that if $\mu$ satisfies a doubling condition together with a local Poincaré inequality, then (1.7) holds true, for all $t$ and for all $x$ outside a set $N_{t}$ of $\mu$ measure 0 . Under the geometric assumption that $(X, d)$ is finite dimensional with Aleksandrov curvature bounded from below, Lott and Villani obtained the validity of (1.7) for all $t$ and $x$. In Theorem 22.46 of [20], Villani proves (1.7) for all $t$ and $x$ on a Riemannian manifold.

We indicate that, during the preparation of this work, we learned that Theorem 1.1 has also been obtained by Ambrosio, Gigli and Savaré in their recent paper [1] (see also [2]), with a very similar proof. Let us underline that the inequality

$$
\frac{d}{d t_{+}} Q_{t} f(x) \leq-\alpha^{*}\left(\left|\nabla^{-} Q_{t} f\right|(x)\right)
$$


which is equivalent to (1.6), is an important ingredient in their study of gradient flows of entropic functionals over general metric spaces. The main source of inspiration for the present paper is the seminal work by Bobkov, Gentil and Ledoux in [4] establishing the equivalence between the logarithmic Sobolev inequality and hypercontractivity properties of Hamilton-Jacobi solutions.

The main tool in the proof of Theorem 1.1 is the following result of independent interest.

Theorem 1.2. Let $f: X \rightarrow \mathbb{R}$ be an upper semicontinuous function bounded from above. For all $t>0$ and $x \in X$, denote by $m(t, x)$ the set of points where the supremum (1.3) defining $P_{t} f(x)$ is reached:

$$
m(t, x)=\left\{\bar{y} \in X: P_{t} f(x)=f(\bar{y})-t \alpha\left(\frac{d(x, \bar{y})}{t}\right)\right\} .
$$

These sets are always non empty and compact and there hold

$$
\frac{d}{d t_{+}} P_{t} f(x)=\beta\left(\frac{1}{t} \max _{\bar{y} \in m(t, x)} d(x, \bar{y})\right), \quad \forall t>0, \quad \forall x \in X
$$

and

$$
\frac{d}{d t_{-}} P_{t} f(x)=\beta\left(\frac{1}{t} \min _{\bar{y} \in m(t, x)} d(x, \bar{y})\right), \quad \forall t>0, \quad \forall x \in X,
$$

where $\beta(h)=h \alpha^{\prime}(h)-\alpha(h), h \geq 0$.

\subsection{Hypercontractivity of $Q_{t}$ and the log-Sobolev inequality}

Let $\mu$ be a Borel probability measure on $X$. Recall that the entropy functional $\operatorname{Ent}_{\mu}(\cdot)$ is defined by

$$
\operatorname{Ent}_{\mu}(g)=\int g \log \left(\frac{g}{\int g d \mu}\right) d \mu, \quad \forall g>0 .
$$

In order to introduce the log-Sobolev inequality, and for technical reasons, define, for $r>0$,

$$
\operatorname{Lip}(f, r)=\sup _{\substack{x, y: \\ d(x, y) \leq r}} \frac{|f(y)-d(x)|}{d(x, y)},
$$

and observe that the usual Lipschitz constant is $\operatorname{Lip}(f)=\sup _{r} \operatorname{Lip}(f, r)$. Then, we denote by $\mathcal{F}_{\alpha}$ the set of bounded functions $f: X \rightarrow \mathbb{R}$ such that $\operatorname{Lip}(f, r)<\infty$ for some $r>0$ and

$$
\operatorname{Lip}(f) \leq \lim _{h \rightarrow \infty} \frac{\alpha(h)}{h}
$$

(observe that if $\alpha(h) / h \rightarrow \infty$ when $h \rightarrow \infty$, this last condition is empty).

The probability measure $\mu$ is said to satisfy the modified log-Sobolev inequality minus $\mathbf{L S I}_{\alpha}^{-}(C)$ for some $C>0$ if

$$
\left(\mathbf{L S I}_{\alpha}^{-}(C)\right) \quad \operatorname{Ent}_{\mu}\left(e^{f}\right) \leq C \int \alpha^{*}\left(\left|\nabla^{-} f\right|\right) e^{f} d \mu \quad \forall f \in \mathcal{F}_{\alpha} .
$$


In particular, when $\alpha(h)=h^{p} / p, h \geq 0$, with $p>1$, there holds $\alpha^{*}(h)=h^{q} / q$, $h \geq 0$ with $1 / p+1 / q=1$. In this case, we write $\mathbf{L S I}_{q}^{-}$for $\mathbf{L S I}_{\alpha}^{-}$. If $X$ is a Riemannian manifold and $\mu$ is absolutely continuous with respect to the volume element, the inequality $\mathbf{L S I}_{2}^{-}$is the usual logarithmic Sobolev inequality introduced by Gross [13].

Following Bobkov, Gentil and Ledoux, [4], we relate $\mathbf{L S I}_{\alpha}^{-}(C)$ to hypercontractivity properties of the family of operators $\left\{Q_{t}\right\}_{t>0}$. To perform the proof, we need to make some restrictions on the function $\alpha$. We will say that $\alpha$ satisfies the $\Delta_{2}$-condition, [17], if there is a positive constant $K$ such that

$$
\alpha(2 x) \leq K \alpha(x), \quad \forall x \geq 0
$$

Theorem 1.3. Suppose that $\alpha$ satisfies the $\Delta_{2}$-condition. Then the exponents $r_{\alpha} \leq p_{\alpha}$ defined by

$$
r_{\alpha}=\inf _{x>0} \frac{x \alpha^{\prime}(x)}{\alpha(x)} \geq 1 \quad \text { and } \quad 1<p_{\alpha}=\sup _{x>0} \frac{x \alpha^{\prime}(x)}{\alpha(x)}
$$

are both finite. Moreover, the measure $\mu$ satisfies $\mathbf{L S I}_{\alpha}^{-}(C)$ if and only if for all $t>0$, for all $t_{o} \leq C\left(p_{\alpha}-1\right)$ and for all bounded continuous functions $f: X \rightarrow \mathbb{R}$,

$$
\left\|e^{Q_{t} f}\right\|_{k(t)} \leq\left\|e^{f}\right\|_{k(0)},
$$

with

$$
k(t)= \begin{cases}\left(1+\frac{C^{-1}\left(t-t_{o}\right)}{p_{\alpha}-1}\right)^{p_{\alpha}-1} \mathbf{1}_{t \leq t_{o}}+\left(1+\frac{C^{-1}\left(t-t_{o}\right)}{r_{\alpha}-1}\right)^{r_{\alpha}-1} \mathbf{1}_{t>t_{o}} & \text { if } r_{\alpha}>1 \\ \min \left(1 ;\left(1+\frac{C^{-1}\left(t-t_{o}\right)}{p_{\alpha}-1}\right)^{p_{\alpha}-1}\right) & \text { if } r_{\alpha}=1\end{cases}
$$

where $\|g\|_{k}=\left(\int|g|^{k} d \mu\right)^{1 / k}$ for $k \neq 0$ and $\|g\|_{0}=\exp \left(\int \log g d \mu\right)$.

Our proof follows the lines of [4]. Let us explain in few words how to derive (1.9) from $\mathbf{L S I}_{\alpha}^{-}$. Since $Q_{t} f \rightarrow f$ when $t \rightarrow 0$, it is enough to show that $H: t \mapsto \log \left\|e^{Q_{t} f}\right\|_{k(t)}$ is non-increasing. The left derivative of $H$ has an expression involving $\operatorname{Ent}_{\mu}\left(e^{k(t) Q_{t} f}\right)$ and $\int \frac{d}{d t_{+}} Q_{t} f e^{k(t) Q_{t} f} d \mu$ (see Proposition 4.1). To bound the first term from above, we apply the inequality $\mathbf{L S I}_{\alpha}^{-}$. To bound the second term, we use the inequality (1.8) which is precisely in the right direction to prove that the left derivative of $H$ is negative.

\subsection{From log-Sobolev to transport-entropy inequalities}

Following [4] and [14], a by-product of the above hypercontractivity result is a metric space extension of Otto-Villani's theorem [16] that indicates that log-Sobolev inequalities imply transport-entropy inequalities.

Let $c: X \times X \rightarrow \mathbb{R}$ be a continuous function; recall that the optimal transport $\operatorname{cost} \mathcal{T}_{c}\left(\nu_{1}, \nu_{2}\right)$ between two Borel probability measures $\nu_{1}, \nu_{2} \in \mathcal{P}(X)$ (the set of 
all Borel probability measures on $X$ ) is defined by

$$
\mathcal{T}_{c}\left(\nu_{1}, \nu_{2}\right)=\inf _{\pi \in P\left(\nu_{1}, \nu_{2}\right)} \iint c(x, y) \pi(d x d y)
$$

where $P\left(\nu_{1}, \nu_{2}\right)$ is the set of all probability measures $\pi$ on $X \times X$ such that $\pi(d x \times$ $X)=\nu_{1}(d x)$ and $\pi(X \times d y)=\nu_{2}(d y)$.

The probability measure $\mu$ is said to satisfy the transport-entropy inequality $\mathbf{T}_{c}(C)$ for some $C>0$ if

$$
\mathcal{T}_{c}(\mu, \nu) \leq C H(\nu \mid \mu), \quad \forall \nu \in \mathcal{P}(X),
$$

where

$$
H(\nu \mid \mu)= \begin{cases}\int \log \frac{d \nu}{d \mu} d \nu & \text { if } \nu \ll \mu, \\ +\infty & \text { otherwise, }\end{cases}
$$

is the relative entropy of $\nu$ with respect to $\mu$. This class of inequalities was introduced by Marton and Talagrand, [15], [19]. When $c(x, y)=\alpha(d(x, y))$ we denote the optimal transport cost by $\mathcal{T}_{\alpha}(\cdot, \cdot)$ and the corresponding transport inequality by $\mathbf{T}_{\alpha}$. In the particular case when $\alpha(x)=x^{p} / p, p \geq 2$ we use the notation $\mathcal{T}_{p}$ and $\mathbf{T}_{p}$.

The first point of the next theorem will appear to be an easy consequence of Theorem 1.3 and of Bobkov and Götze's dual formulation of the inequality $\mathbf{T}_{\alpha}$ (which roughly speaking corresponds to the hypercontractivity with $t_{o}=C\left(p_{\alpha}-1\right)$ or equivalently $k(0)=0)$.

Theorem 1.4. Suppose that $\alpha$ satisfies the $\Delta_{2}$-condition. If $\mu$ satisfies $\mathbf{L S I}_{\alpha}^{-}(C)$, then it satisfies $\mathbf{T}_{\alpha}(A)$, with

$$
A=\max \left(\left(\left(p_{\alpha}-1\right) C\right)^{r_{\alpha}-1} ;\left(\left(p_{\alpha}-1\right) C\right)^{p_{\alpha}-1}\right),
$$

where the numbers $r_{\alpha}$ and $p_{\alpha}$ are defined in Theorem 1.3.

In a Riemannian framework and for the quadratic function $\alpha(t)=t^{2} / 2$, Theorem 1.4 was first obtained by Otto and Villani in [16], closely followed by Bobkov, Gentil and Ledoux [4]. Extensions to other functions $\alpha$ were provided in [4] and [8]. The path space case was treated by Wang in [21]. In [14], Lott and Villani extended to certain geodesic measured spaces $(X, d, \mu)$ the Hamilton-Jacobi approach of [4] in the quadratic case. They proved Theorem 1.4 under additional assumptions on $\mu$ (doubling property and local Poincaré). Under the same assumptions Balogh, Engoulatov, Hunziker and Maasalo [3] treated the case of $\mathbf{L S I}_{q}^{-}$for all $q \leq 2$. The first proofs of the Otto-Villani theorem valid on any complete separable metric space appeared in [10] and [12]. Their common feature is the use of the stability of the log-Sobolev inequality under tensor products of the reference probability measure. In a recent paper [9], Gigli and Ledoux give another quick proof of the Otto-Villani theorem on metric spaces. It is based on calculations along gradient flows in the Wasserstein space.

Using some rough properties of the operators $Q_{t}$, we also provide a metric space generalization of another result by Otto and Villani ([16]) relating transportentropy inequalities to the Poincaré inequality. 
Proposition 1.5. Let $\theta: \mathbb{R}^{+} \rightarrow \mathbb{R}^{+}$be any function such that $\theta(x) \geq \min \left(x^{2}, a^{2}\right)$ for some $a>0$. If $\mu$ satisfies $\mathbf{T}_{\theta}(C)$ for some $C>0$, then it satisfies the following Poincaré inequality:

$$
\operatorname{Var}_{\mu}(f) \leq \frac{C}{2} \int\left|\nabla^{-} f\right|^{2} d \mu,
$$

for all bounded function $f$ such that $\operatorname{Lip}(f, r)<\infty$, for some $r>0$.

\subsection{Transport-entropy inequalities as restricted log-Sobolev inequali- ties}

A second consequence of the Hamilton-Jacobi approach on metric spaces is a characterization of transport-entropy inequalities in terms of log-Sobolev inequalities restricted to a certain class of functions depending on the cost function $\alpha$.

To be more precise, let us say that a function $f$ is $c$-convex with respect to a cost function $(x, y) \mapsto c(x, y)$ defined on $X \times X$ if there is a function $g: X \rightarrow \mathbb{R} \cup\{ \pm \infty\}$ such that

$$
f(x)=P_{c} g(x)=\sup _{y \in X}\{g(y)-c(x, y)\} \in \mathbb{R} \cup\{ \pm \infty\}, \quad \forall x \in X .
$$

The class of $c$-convex functions is intimately related to optimal-transport, via for instance the Kantorovich duality theorem (see e.g. [20]).

An important case is when $c(x, y)=\frac{1}{2}\|x-y\|_{2}^{2}$ on $\mathbb{R}^{m}$ (see Proposition 2.3 below). In this case, a function $f: \mathbb{R}^{m} \rightarrow \mathbb{R}$ is $c$-convex if and only if the function $x \mapsto f(x)+\|x\|_{2}^{2} / 2$ is convex on $\mathbb{R}^{m}$. If $f$ is of class $\mathcal{C}^{2}$, this amounts to saying that Hess $f \geq-$ Id.

In what follows, we consider the cost $c_{p}(x, y)=d^{p}(x, y) / p, p \geq 2$. The second main result of this paper is the following.

Theorem 1.6. Let $\mu$ be a probability measure on a geodesic space $(X, d)$ and $p \geq 2$. The following properties are equivalent:

1. There is some $C>0$ such that $\mu$ satisfies $\mathbf{T}_{p}(C)$.

2. There is some $D>0$ such that $\mu$ satisfies the following $(\tau)$-log-Sobolev inequality: for all bounded continuous $f$ and all $0<\lambda<1 / D$, there holds

$$
\operatorname{Ent}_{\mu}\left(e^{f}\right) \leq \frac{1}{1-\lambda D} \int\left(f-Q^{\lambda} f\right) e^{f} d \mu,
$$

where for all $\lambda>0, Q^{\lambda} f(x)=\inf _{y \in X}\left\{f(y)+\lambda c_{p}(x, y)\right\}$.

3. There is some $E>0$ such that $\mu$ satisfies the following restricted log-Sobolev inequality: for all $K c_{p}$-convex function $f$, with $0<K<1 / E$ there holds

$$
\operatorname{Ent}_{\mu}\left(e^{f}\right) \leq \frac{\beta_{p}(u)-1}{p K^{q-1}(1-K E u)} \int\left|\nabla^{+} f\right|^{q} e^{f} d \mu, \quad \forall u \in(1,1 /(K E)),
$$

where $q=p /(p-1)$ and $\beta_{p}(u)=\frac{u}{\left[u^{1 /(p-1)}-1\right]^{p-1}}$ for all $u>1$. 
The optimal constants $C_{\mathrm{opt}}, D_{\mathrm{opt}}$ and $E_{\mathrm{opt}}$ are related by

$$
E_{\mathrm{opt}} \leq D_{\mathrm{opt}} \leq C_{\mathrm{opt}} \leq \kappa_{p} E_{\mathrm{opt}}
$$

where $\kappa_{p}$ is some universal constant depending only on $p$. For $p=2$, one can take $\kappa_{2}=e^{2}$.

We make some comments on Theorem 1.6.

- The first reason why we suppose $p \geq 2$ in Theorem 1.6 (as well as in Theorems 5.1 and 5.5 and Proposition 5.4), is that the only probability measures that satisfy the transport inequality associated to the cost function $c_{p}(x, y)=d^{p}(x, y) / p$ for $0<p<2$ are Dirac measures (see Remark 1.3 in [12]). The second reason, is that the notation $\mathbf{T}_{1}$ is classically used in the literature to indicate the transport inequality

$$
\mathcal{T}_{1}(\nu, \mu) \leq \sqrt{C H(\nu \mid \mu)}, \quad \forall \nu \in \mathcal{P}(X) .
$$

This has been characterized by Djelout, Guillin and Wu in [6] by an integrability condition of the form $\iint e^{\varepsilon d^{2}(x, y)} \mu(d x) \mu(d y)<+\infty$, for some $\varepsilon>0$.

- The implication $(1) \Rightarrow(2)$ is true for any cost function $c$. This was first proved in [11].

- In [12], we proved that (1) is equivalent to (2) for cost functions $c(x, y)=$ $\alpha(d(x, y))$ as soon as $\alpha$ satisfies the $\Delta_{2}$-condition. Our proof (in [12]) makes use of a tensorization technique and is thus rather different from the one presented here.

- In [11], we proved the equivalence between (1), (2) and (3) in a Euclidean framework: $X=\mathbb{R}^{m}$ and $c(x, y)=\frac{1}{2}\|x-y\|_{2}^{2}$. Actually, the result of [11] is slightly more general, since we are able to deal with cost functions of the form $c(x, y)=\sum_{i=1}^{m} \theta\left(\left|x_{i}-y_{i}\right|\right), x, y \in \mathbb{R}^{m}$, where $\theta: \mathbb{R}^{+} \rightarrow \mathbb{R}^{+}$is a convex function of class $\mathcal{C}^{1}$ such that $\theta(0)=\theta^{\prime}(0)=0$ and $\theta^{\prime}$ is concave on $\mathbb{R}^{+}$. For a cost function of this type, we proved that (1) and (2) are both equivalent to the following restricted modified log-Sobolev inequality (3') (see Theorem 1.5 in [11] for a precise statement): there are $E, K>0$ such that

$$
\operatorname{Ent}_{\mu}\left(e^{f}\right) \leq E \int \sum_{i=1}^{m} \theta^{*}\left(\partial_{i} f\right) e^{f} d \mu,
$$

for all functions $f: \mathbb{R}^{m} \rightarrow \mathbb{R}$ of class $\mathcal{C}^{1}$ which are semiconvex in the sense that

$$
f(y) \geq f(x)+\nabla f(x) \cdot(y-x)-K c(x, y), \quad \forall x, y \in \mathbb{R}^{m} .
$$

Note that this class of functions is different from the class of $K c$-convex functions (except when $c(x, y)=\frac{1}{2}\|x-y\|_{2}^{2}$; see Proposition 2.3 below). Note also that a function $\theta$ satisfying the condition above necessarily satisfies $\theta(t) \leq \theta^{\prime \prime}(0) t^{2} / 2, t \geq 0$. In particular, the cost function $c(x, y)=\frac{1}{p}\|x-y\|_{p}^{p}$, for $p>2$ is not in the scope of [11], whereas Theorem 1.6 of the present paper enables to consider such a cost function. 
Theorem 1.6 thus provides what we think is a good extension of the results in [11] and unifies nicely the approaches of [11] and [12].

We mention that Theorem 1.6 as stated above is not as general as possible. Indeed, we will see in Section 5 that this equivalence is still true when the space is not geodesic (Theorem 5.1). In this more general framework, (3) has to be replaced by a slightly weaker version of the restricted log-Sobolev inequality. The main tool to prove this extension is Theorem 1.2. It would also be possible to consider more general costs of the form $c(x, y)=\alpha(d(x, y))$ with $\alpha$ satisfying the $\Delta_{2}$-condition but, to avoid some lengthy developments, this will not be treated here.

We end this introduction with a short roadmap of the paper. Section 2 is devoted to $c$-convex functions. In particular, we will recall and prove some well known facts about the subdifferential $\partial_{c} f(x)$ of a $c$-convex function. In Proposition 2.9, we will relate their gradients $\left|\nabla^{ \pm} f\right|(x)$ to the minimal or maximal distance between $x$ and the subdifferential $\partial_{c} f(x)$. Section 3 contains the proof of the HLO formula. In Section 4, we prove the hypercontractivity property of Theorem 1.9, and deduce as a corollary the Otto-Villani Theorem 1.4. Section 5 contains the proof of an improved version of our main result, Theorem 1.6. Finally, the appendix contains some technical results.

\section{About c-convex functions}

In this section we introduce the somehow classical notions of $c$-convex (and $c$-concave) functions and of $c$-subdifferential. We will also give several useful facts about these notions. The interested reader may find more results and comments, and some bibliographic notes, in Chapter 5 of [20].

\subsection{Definition of $c$-convex functions and preliminary results}

Let $X$ and $Y$ be two Polish spaces, let $c: X \times Y \rightarrow \mathbb{R}$ be a general cost function, and set $\overline{\mathbb{R}}=\mathbb{R} \cup\{ \pm \infty\}$. For any function $f: X \rightarrow \overline{\mathbb{R}}$, we define $Q_{c} f: Y \rightarrow \overline{\mathbb{R}}$ by

$$
Q_{c} f(y):=\inf _{x \in X}\{f(x)+c(x, y)\} .
$$

For any function $g: Y \rightarrow \overline{\mathbb{R}}$, we define $P_{c} g: X \rightarrow \overline{\mathbb{R}}$, by

$$
P_{c} g(x):=\sup _{y \in Y}\{g(y)-c(x, y)\}
$$

Definition 2.1 (c-convex and c-concave functions). A function $f: X \rightarrow \overline{\mathbb{R}}$ is said to be $c$-convex if there is some function $g: Y \rightarrow \overline{\mathbb{R}}$ such that $f=P_{c} g$. A function $g: Y \rightarrow \overline{\mathbb{R}}$ is said to be $c$-concave if there is some function $f: X \rightarrow \overline{\mathbb{R}}$ such that $g=Q_{c} f$.

Proposition 2.2. For any function $f: X \rightarrow \overline{\mathbb{R}}$, the inequality $P_{c} Q_{c} f \leq f$ holds. Moreover, $f: X \rightarrow \overline{\mathbb{R}}$ is c-convex if and only if $P_{c} Q_{c} f=f$. 
Proof. For the first point observe that, for $z=x$,

$$
P_{c} Q_{c} f(x)=\sup _{y \in Y} \inf _{z \in X}\{f(z)+c(z, y)-c(x, y)\} \leq f(x) .
$$

Let us prove the second point. Trivially, a function $f$ such that $f=P_{c} Q_{c} f$ is $c$-convex. Conversely, if $f: X \rightarrow \overline{\mathbb{R}}$ is $c$-convex, then there is some function $g$ on $Y$ such that $f(x)=\sup _{y \in Y}\{g(y)-c(x, y)\}=P_{c} g(y)$. Hence $g$ satisfies $g(y) \leq$ $\inf _{x \in X}\{f(x)+c(x, y)\}$. Plugging this inequality into $f=P_{c} g$ gives $f \leq P_{c} Q_{c} f$. Since the other direction always holds, the proof is complete.

Recall that a function $f: \mathbb{R}^{m} \rightarrow \overline{\mathbb{R}}$ is said to be closed (see [18]) if either $f=-\infty$ everywhere or $f$ takes its values in $\mathbb{R} \cup\{+\infty\}$ and is lower semicontinuous. It is said to be convex if its epigraph $\left\{(x, \alpha) \in \mathbb{R}^{m} \times \mathbb{R}: \alpha \geq f(x)\right\}$ is a convex subset of $\mathbb{R}^{m} \times \mathbb{R}$. Let us denote by $\Gamma\left(\mathbb{R}^{m}\right)$ the set of all closed and convex functions on $\mathbb{R}^{m}$.

Proposition 2.3 (Examples). Assume that $X=Y=\mathbb{R}^{m}, m \in \mathbb{N}^{*}$, is equipped with its standard Euclidean structure and let $f: \mathbb{R}^{m} \rightarrow \overline{\mathbb{R}}$. Then:

(1) If $c(x, y)=x \cdot y, f$ is c-convex if and only if $f \in \Gamma\left(\mathbb{R}^{m}\right)$.

(2) If $c(x, y)=\frac{1}{2}\|x-y\|_{2}^{2}, f$ is c-convex if and only if $f+\|\cdot\|_{2}^{2} / 2 \in \Gamma\left(\mathbb{R}^{m}\right)$. In particular, if $f: \mathbb{R}^{m} \rightarrow \mathbb{R}$ is of class $\mathcal{C}^{2}$ then it is c-convex if and only if Hess $f(x) \geq-\mathrm{Id}$, for all $x \in \mathbb{R}^{m}$.

Proof. (1) By definition, a function $f$ is $c$-convex for $c(x, y)=x \cdot y$ if and only if $f=h^{*}$ for some function $h: \mathbb{R}^{m} \rightarrow \overline{\mathbb{R}}$. We recall that $h^{*}$ is defined by $h^{*}(x)=$ $\sup _{y \in \mathbb{R}^{m}}\{x \cdot y-h(y)\}$. It is well known (and easy to check) that $h^{*} \in \Gamma\left(\mathbb{R}^{m}\right)$ for all $h$. Conversely, if $f \in \Gamma\left(\mathbb{R}^{m}\right)$ then $f=f^{* *}$ (see e.g [18]) and so $f$ is $c$-convex.

(2) The function $f$ is a $c$-convex function for $c(x, y)=\|x-y\|_{2}^{2} / 2$ if and only if $f=P_{c} g$, for some $g: \mathbb{R}^{m} \rightarrow \overline{\mathbb{R}}$. Since

$$
f(x)+\frac{\|x\|_{2}^{2}}{2}=\sup _{y \in \mathbb{R}^{m}}\left\{x \cdot y-\left(\frac{\|y\|_{2}^{2}}{2}-g(y)\right)\right\},
$$

the conclusion follows from the first point.

\subsection{The $c$-subdifferential of a $c$-convex function}

In this section we define the notion of $c$-subdifferential of a $c$-convex function and derive some facts that will be useful later.

Definition 2.4 (c-subdifferential). Let $f: X \rightarrow \overline{\mathbb{R}}$ be a $c$-convex function and let $x \in X$. The $c$-subdifferential of $f$ at point $x$ is the set, denoted by $\partial_{c} f(x) \subset Y$, of points $\bar{y} \in Y$ such that

$$
f(z) \geq f(x)+c(x, \bar{y})-c(z, \bar{y}), \quad \forall z \in X .
$$

The next lemma gives a characterization of the $c$-subdifferential. 
Lemma 2.5. For all $x \in X, \partial_{c} f(x)$ is the set of points $y \in Y$ attaining the supremum in $f(x)=P_{c} Q_{c} f(x)$. More precisely,

$$
\partial_{c} f(x)=\left\{y \in Y: f(x)=Q_{c} f(y)-c(x, y)\right\} .
$$

More generally, if $f=P_{c} g$, for some function $g: Y \rightarrow \overline{\mathbb{R}}$, then

$$
\{y \in Y: f(x)=g(y)-c(x, y)\} \subset \partial_{c} f(x) .
$$

Proof. The first part of the lemma is simple and is left to the reader. Let us prove the second part. Since $f(x)=\sup _{y \in Y}\{g(y)-c(x, y)\}, x \in X$, we have $g \leq Q_{c} f$. So if, $f(x)=g(\bar{y})-c(x, \bar{y})$ then $f(x) \leq Q_{c} f(\bar{y})-c(x, \bar{y}) \leq f(z)+c(z, \bar{y})-c(x, \bar{y})$, for all $z \in X$ which proves that $\bar{y} \in \partial_{c} f(x)$.

Lemma 2.6. Suppose that the function $c: X \times Y \rightarrow \mathbb{R}$ is continuous, satisfies $\sup _{v \in Y} \inf _{u \in X} c(u, v)<+\infty$ and that, for all $x \in X$ and $r \in \mathbb{R}$, the level sets $\{y \in Y ; c(x, y) \leq r\}$ are compact. If $f: X \rightarrow \mathbb{R} \cup\{-\infty\}$ is a c-convex function bounded from above, then $\partial_{c} f(x) \neq \emptyset$ for all $x \in X$.

Remark 2.7. Note that, when $X=Y$, the condition $\sup _{v \in X} \inf _{u \in X} c(u, v)<+\infty$ is always satisfied if $c(x, y)=\alpha(d(x, y))$, where $d$ is a distance on $X$ and $\alpha: \mathbb{R}^{+} \rightarrow \mathbb{R}$ a non-decreasing function.

Proof. The function $Q_{c} f$ is an infimum of continuous functions on $Y$, so it is upper semicontinuous on $Y$. For all $x \in X$, the function $\varphi_{x}: y \mapsto Q_{c} f(y)-c(x, y)$ is thus upper semicontinuous on $Y$. Since $f$ is $c$-convex and real valued, $\sup _{y \in Y} \varphi_{x}(y)=$ $P_{c} Q_{c} f(x)=f(x)<+\infty$; so $\varphi_{x}$ is bounded from above. Finally if $y \in\left\{\varphi_{x} \geq r\right\}$ then $c(x, y) \leq \sup f+\sup _{v \in Y} \inf _{u \in X} c(u, v)-r$. Hence $\left\{\varphi_{x} \geq r\right\}$ is compact. From this it follows that $\varphi_{x}$ achieves its supremum at some point $\bar{y}$ which, according to Lemma 2.5, necessarily belongs to $\partial_{c} f(x)$.

For a better understanding of the notion, in the next lemma we express the $c$-subdifferential of a $c$-convex function $f$ in term of its gradient in some simple cases.

Lemma 2.8. Suppose that $X=Y=\mathbb{R}^{m}$ and that $c(x, y)=L(x-y)$ where $L: \mathbb{R}^{m} \rightarrow \mathbb{R}^{+}$is a differentiable and strictly convex function with superlinear growth, i.e., $L(x) /\|x\| \rightarrow+\infty$ when $x \rightarrow \infty$, where $\|\cdot\|$ denotes any norm on $\mathbb{R}^{m}$. Let $f$ be a c-convex function bounded from above and differentiable at some point $x$. Then

$$
\partial_{c} f(x)=\left\{x-\nabla\left(L^{*}\right)(-\nabla f(x))\right\},
$$

where $L^{*}(y)=\sup _{x \in \mathbb{R}^{m}}\{x \cdot y-L(y)\}$ is the Fenchel-Legendre transform of $L$.

We recall that if $L$ is strictly convex and has superlinear growth, then its Fenchel-Legendre transform is differentiable everywhere [18]. Lemma 2.8 is well known. However, for the sake of completeness, we will recall its proof in the appendix. 


\subsection{Comparisons of gradients}

In this last section, as in the rest of the paper, we will assume that $(X, d)$ is a complete separable metric space in which closed balls are compact. We take $Y=X$ and we consider a cost function $c$ on $X \times X$ of the form

$$
c(x, y)=\alpha(d(x, y))
$$

where $\alpha: \mathbb{R}^{+} \rightarrow \mathbb{R}^{+}$is an increasing convex function of class $\mathcal{C}^{1}$ such that $\alpha(0)=0$.

If $f: X \rightarrow \mathbb{R}$ is $c$-convex for the cost $c(x, y)=\alpha(d(x, y))$, we introduce the following quantities:

$$
\left|\nabla_{c}^{-} f\right|(x)=\alpha^{\prime}\left(\inf _{\bar{y} \in \partial_{c} f(x)} d(x, \bar{y})\right) \quad \text { and } \quad\left|\nabla_{c}^{+} f\right|(x)=\alpha^{\prime}\left(\sup _{\bar{y} \in \partial_{c} f(x)} d(x, \bar{y})\right) .
$$

The following proposition compares $\left|\nabla_{c}^{ \pm} f\right|$ to $\left|\nabla^{ \pm} f\right|$ defined in (1.5).

Proposition 2.9. Let $f: X \rightarrow \mathbb{R}$ be a c-convex function for the cost $c(x, y)=$ $\alpha(d(x, y))$. Suppose that $f=P_{c} g$ for some upper semicontinuous function $g$ : $X \rightarrow \mathbb{R}$ bounded from above, and consider, for all $x \in X$, the set $m(x)$ defined by $m(x)=\{y \in X: f(x)=g(y)-\alpha(d(x, y))\}$.

(1) The following inequalities hold:

$$
\left|\nabla^{+} f\right|(x) \leq \alpha^{\prime}\left(\max _{\bar{y} \in m(x)} d(x, \bar{y})\right) \leq\left|\nabla_{c}^{+} f\right|(x) .
$$

(2) If $(X, d)$ is a geodesic space, then

$$
\left|\nabla^{+} f\right|(x)=\alpha^{\prime}\left(\max _{\bar{y} \in m(x)} d(x, \bar{y})\right)=\left|\nabla_{c}^{+} f\right|(x) .
$$

(3) The following inequalities hold:

$$
\left|\nabla^{-} f\right|(x) \leq\left|\nabla_{c}^{-} f\right|(x) \leq \alpha^{\prime}\left(\min _{\bar{y} \in m(x)} d(x, \bar{y})\right) .
$$

Remark 2.10. We do not know if there is equality in (3) when the space is geodesic.

Proof of Proposition 2.9. (1) First observe that, since $f=P_{c} g$ with $g$ bounded above, $f$ is locally Lipschitz (see Lemma 3.8 in $[12])$ ), so that $\left|\nabla^{+} f\right|$ is finite everywhere. The second inequality is an immediate consequence of the definition of $\left|\nabla_{c}^{+} f\right|(x)$ and the fact that, according to Lemma 2.5, $m(x) \subset \partial_{c} f(x)$. Let us prove the first inequality. Let $\left(x_{n}\right)_{n \in \mathbb{N}}$ be a sequence of points converging to $x$, with $x_{n} \neq x$ for all $n$. For all $n$, fix $y_{n} \in m\left(x_{n}\right)$ (the set $m\left(x_{n}\right)$ is not empty, by Lemma 2.11 below). There holds

$$
\begin{aligned}
f\left(x_{n}\right)-f(x) & \leq g\left(y_{n}\right)-\alpha\left(d\left(x_{n}, y_{n}\right)\right)-\left(g\left(y_{n}\right)-\alpha\left(d\left(x, y_{n}\right)\right)\right) \\
& \leq d\left(x, x_{n}\right) \alpha^{\prime}\left(\max \left(d\left(x_{n}, y_{n}\right) ; d\left(x, y_{n}\right)\right)\right),
\end{aligned}
$$


where the last inequality follows from the mean value theorem, the triangle inequality, and the non-negativity and monotonicity of $\alpha^{\prime}$. From this it follows that

$$
\frac{\left[f\left(x_{n}\right)-f(x)\right]_{+}}{d\left(x_{n}, x\right)} \leq \alpha^{\prime}\left(\max \left(d\left(x_{n}, y_{n}\right) ; d\left(x, y_{n}\right)\right)\right)
$$

So, letting $n \rightarrow \infty$,

$$
\begin{aligned}
& \limsup _{n \rightarrow \infty} \frac{\left[f\left(x_{n}\right)-f(x)\right]_{+}}{d\left(x_{n}, x\right)} \leq \alpha^{\prime}\left(\limsup _{n \rightarrow \infty} d\left(x, y_{n}\right)\right) \\
& \quad=\alpha^{\prime}\left(\max \left\{d(x, \bar{y}): \bar{y} \text { limit point of }\left(y_{n}\right)_{n \in \mathbb{N}}\right\}\right) \leq \alpha^{\prime}\left(\max _{\bar{y} \in m(x)} d(x, \bar{y})\right),
\end{aligned}
$$

where the last inequality follows from Lemma 2.11 below.

(2) To prove the second point it is enough to show that $\left|\nabla_{c}^{+} f\right|(x) \leq\left|\nabla^{+} f\right|(x)$ for all $x \in X$. Let $\bar{y} \in \partial_{c} f(x)$ (this set is not empty, by Lemma 2.6). According to the definition of the $c$-subdifferential,

$$
f(z)-f(x) \geq \alpha(d(x, \bar{y}))-\alpha(d(z, \bar{y})), \quad \forall z \in X .
$$

From the definition of $\left|\nabla^{+} f\right|(x)$, it follows that

$$
\left|\nabla^{+} f\right|(x) \geq \limsup _{z \rightarrow x} \frac{\alpha(d(x, \bar{y}))-\alpha(d(z, \bar{y}))}{d(x, z)} .
$$

Let $\left(z_{t}\right)_{t \in[0,1]}$ be a geodesic connecting $x$ to $\bar{y}$, there holds $d\left(x, z_{t}\right)=t d(x, \bar{y})$, $d\left(z_{t}, \bar{y}\right)=(1-t) d(x, \bar{y})$ and therefore

$$
\left|\nabla^{+} f\right|(x) \geq \limsup _{t \rightarrow 0} \frac{\alpha(d(x, \bar{y}))-\alpha((1-t) d(x, \bar{y}))}{t d(x, \bar{y})}=\alpha^{\prime}(d(x, \bar{y})) .
$$

Optimizing over all $\bar{y} \in \partial_{c} f(x)$ completes the proof.

(3) Let $\left(x_{n}\right)_{n \in \mathbb{N}}$ be a sequence of points converging to $x$, with $x_{n} \neq x$ for all $n$. If $\bar{y} \in \partial_{c} f(x)$, then there holds

$$
f\left(x_{n}\right)-f(x) \geq \alpha(d(x, \bar{y}))-\alpha\left(d\left(x_{n}, \bar{y}\right)\right) \geq-d\left(x, x_{n}\right) \alpha^{\prime}\left(\max \left(d\left(x_{n}, \bar{y}\right) ; d(x, \bar{y})\right)\right),
$$

where the second inequality follows from the mean value theorem and the triangle inequality. From this it easily follows that

$$
\limsup _{n \rightarrow+\infty} \frac{\left[f\left(x_{n}\right)-f(x)\right]_{-}}{d\left(x, z_{n}\right)} \leq \alpha^{\prime}(d(x, \bar{y})) .
$$

Optimizing over all $\bar{y} \in \partial_{c} f(x)$ leads to the first bound in (3). As above, the second inequality in (3) is an immediate consequence of the definition of $\left|\nabla_{c}^{-} f\right|(x)$ together with the fact that, by Lemma 2.5, $m(x) \subset \partial_{c} f(x)$. This completes the proof.

During the proof we have used the following simple lemma whose proof can be found in the appendix. 
Lemma 2.11. Let $X$ be a complete separable metric space with compact balls and let $g: X \rightarrow \mathbb{R}$ be an upper semicontinuous function bounded from above. Define, for all $x \in X, P_{t} g(x)=\sup _{y \in X}\{g(y)-t \alpha(d(x, y) / t)\}$ and let $m(t, x)$ be the set of points $y \in X$ where this supremum is attained. Then:

(1) The set $m(t, x)$ is a non empty compact set of $X$.

(2) Let $x_{n} \rightarrow x \in X$ and $t_{n} \rightarrow t>0$ be two converging sequences and consider a sequence $\left(y_{n}\right)_{n \in \mathbb{N}}$ such that $y_{n} \in m\left(t_{n}, x_{n}\right)$ for all $n$. Then $\left(y_{n}\right)_{n \in \mathbb{N}}$ is bounded and all its limit points belong to $m(t, x)$.

\section{Proof of the Hamilton-Jacobi equations}

This part is devoted to the proof of Theorems 1.1 and 1.2.

Proof of Theorem 1.2. By Lemma 2.11, $m(t, x)$ is a non empty compact set of $X$. We treat the case of the right derivative; the other case is completely analogous. Let $t>0$ and $x \in X$, and let $\left(h_{n}\right)_{n \in \mathbb{N}}$ be a sequence of positive numbers converging to 0 . For all $n \in \mathbb{N}$, we consider $z_{n} \in m\left(t+h_{n}, x\right)$. Then,

$$
\begin{aligned}
\frac{1}{h_{n}}\left(P_{t+h_{n}}\right. & \left.f(x)-P_{t} f(x)\right) \\
& \leq \frac{1}{h_{n}}\left[f\left(z_{n}\right)-\left(t+h_{n}\right) \alpha\left(\frac{d\left(x, z_{n}\right)}{t+h_{n}}\right)-\left(f\left(z_{n}\right)-t \alpha\left(\frac{d\left(x, z_{n}\right)}{t}\right)\right)\right] \\
& =\frac{1}{h_{n}}\left[t \alpha\left(\frac{d\left(x, z_{n}\right)}{t}\right)-\left(t+h_{n}\right) \alpha\left(\frac{d\left(x, z_{n}\right)}{t+h_{n}}\right)\right] .
\end{aligned}
$$

Define $D=\lim \sup _{k \rightarrow \infty} d\left(x, z_{k}\right)$ and take $\varepsilon>0$. For all $n$ large enough, $d\left(x, z_{n}\right) \leq$ $D+\varepsilon$. For all $h \geq 0$ and all $t>0$, by the convexity assumption on $\alpha$, the map

$$
d \mapsto t \alpha\left(\frac{d}{t}\right)-(t+h) \alpha\left(\frac{d}{t+h}\right)
$$

is non-decreasing. Hence

$$
\begin{aligned}
\limsup _{n \rightarrow \infty} \frac{1}{h_{n}}[t & \left.\alpha\left(\frac{d\left(x, z_{n}\right)}{t}\right)-\left(t+h_{n}\right) \alpha\left(\frac{d\left(x, z_{n}\right)}{t+h_{n}}\right)\right] \\
& \leq \lim _{n \rightarrow \infty} \frac{1}{h_{n}}\left[t \alpha\left(\frac{D+\varepsilon}{t}\right)-\left(t+h_{n}\right) \alpha\left(\frac{D+\varepsilon}{t+h_{n}}\right)\right]=\beta\left(\frac{D+\varepsilon}{t}\right)
\end{aligned}
$$

where we recall that $\beta(h)=h \alpha^{\prime}(h)-\alpha(h), h \geq 0$. Since $\alpha$ is of class $\mathcal{C}^{1}$, as $\varepsilon$ goes to 0 we get

$$
\limsup _{n \rightarrow+\infty} \frac{1}{h_{n}}\left(P_{t+h_{n}} f(x)-P_{t} f(x)\right) \leq \beta\left(\frac{D}{t}\right) .
$$

Applying Lemma 2.11, it is not difficult to check that

$$
D=\limsup _{n \rightarrow \infty} d\left(x, z_{n}\right)=\max \left\{d(x, \bar{z}): \bar{z} \text { limit point of }\left(z_{n}\right)_{n \in \mathbb{N}}\right\} \leq \max _{\bar{y} \in m(t, x)} d(x, \bar{y}) .
$$


The conditions on $\alpha$ ensure that $\beta$ is non-decreasing and therefore

$$
\limsup _{n \rightarrow+\infty} \frac{1}{h_{n}}\left(P_{t+h_{n}} f(x)-P_{t} f(x)\right) \leq \beta\left(\frac{\max _{\bar{y} \in m(t, x)} d(x, \bar{y})}{t}\right) .
$$

Analogously, if $\bar{y} \in m(t, x)$ then

$$
\frac{1}{h_{n}}\left(P_{t+h_{n}} f(x)-P_{t} f(x)\right) \geq \frac{1}{h_{n}}\left(t \alpha\left(\frac{d(x, \bar{y})}{t}\right)-\left(t+h_{n}\right) \alpha\left(\frac{d(x, \bar{y})}{t+h_{n}}\right)\right) .
$$

So, letting $n$ go to $\infty$, and optimizing over $\bar{y}$ yields

$$
\liminf _{n \rightarrow \infty} \frac{1}{h_{n}}\left(P_{t+h_{n}} f(x)-P_{t} f(x)\right) \geq \beta\left(\frac{\max _{\bar{y} \in m(t, x)} d(x, \bar{y})}{t}\right) .
$$

We conclude from (3.1) and (3.2) that

$$
\lim _{n \rightarrow \infty} \frac{1}{h_{n}}\left(P_{t+h_{n}} f(x)-P_{t} f(x)\right)=\beta\left(\frac{\max _{\bar{y} \in m(t, x)} d(x, \bar{y})}{t}\right) .
$$

This completes the proof of Proposition 1.2.

Proof of Theorem 1.1. By Theorem 1.2,

$$
\frac{d}{d t_{+}} P_{t} f(x)=\beta\left(\frac{\max _{\bar{y} \in m(t, x)} d(x, \bar{y})}{t}\right),
$$

with $\beta(u)=u \alpha^{\prime}(u)-\alpha(u)$, for all $u \geq 0$. By the definition of $c$-convexity, the function $x \mapsto P_{t} f(x)$ is $c$-convex for the cost $c(x, y)=t \alpha(d(x, y) / t)$. Applying point (1) of Proposition 2.9, there holds

$$
\left|\nabla^{+} P_{t} f\right|(x) \leq \alpha^{\prime}\left(\frac{\max _{\bar{y} \in m(t, x)} d(x, \bar{y})}{t}\right)
$$

Observing that $\beta(u)=\alpha^{*}\left(\alpha^{\prime}(u)\right)$ gives the result. By point (3) of Proposition 2.9, equality holds in the geodesic case. The proof of the inequality involving the left derivative of $P_{t} f$ is similar.

\section{Log-Sobolev inequality and hypercontractivity on a metric space}

In this section, following [4], we show that log-Sobolev inequalities on metric spaces are equivalent to some hypercontractivity property of the "semigroup" $Q_{t}$. The proof of Theorem 1.3 relies on the differentiation of the left-hand side of (1.9). For this purpose, we use the next technical proposition, whose proof is postponed to the appendix. 
Proposition 4.1. Let $f$ be a bounded and continuous function on $X$ and let $k$ : $(a, b) \rightarrow(0,+\infty)$ be a function of class $\mathcal{C}^{1}$ defined on an open interval $(a, b) \subset$ $(0, \infty)$ and such that $k^{\prime}(t) \neq 0$ for all $t$. Define, for all $t \in(a, b)$,

$$
H(t)=\frac{1}{k(t)} \log \left(\int e^{k(t) Q_{t} f} d \mu\right), \quad \text { and } \quad K(t)=\frac{1}{k(t)} \log \left(\int e^{k(t) P_{t} f} d \mu\right) .
$$

The functions $H$ and $K$ are continuous and differentiable on the right and on the left on $(a, b)$. Moreover, for all $t \in(a, b)$, there holds

$$
\frac{d H}{d t_{+}}(t)=\frac{k^{\prime}(t)}{k(t)^{2}} \frac{1}{\int e^{k(t) Q_{t} f} d \mu}\left[\operatorname{Ent}_{\mu}\left(e^{k(t) Q_{t} f}\right)+\frac{k(t)^{2}}{k^{\prime}(t)} \int\left(\frac{d}{d t_{+}} Q_{t} f\right) e^{k(t) Q_{t} f} d \mu\right] .
$$

The same formula holds for $d H / d t_{-}, d K / d t_{+}$and $d K / d t_{-}$(replacing $Q_{t}$ by $\left.P_{t}\right)$.

Proof of Theorem 1.3. Let us first show that the log-Sobolev inequality implies the hypercontractivity property

$$
\left\|e^{Q_{t} f}\right\|_{k(t)} \leq\left\|e^{f}\right\|_{k(0)}
$$

for all bounded continuous function $f: X \rightarrow \mathbb{R}$, with

$$
k(t)=\left(1+\frac{C^{-1}\left(t-t_{o}\right)}{p_{\alpha}-1}\right)^{p_{\alpha}-1} \mathbf{1}_{t \leq t_{o}}+\left(1+\frac{C^{-1}\left(t-t_{o}\right)}{r_{\alpha}-1}\right)^{r_{\alpha}-1} \mathbf{1}_{t>t_{o}},
$$

with the convention that $k(t)=\min \left(1 ;\left(1+\frac{C^{-1}\left(t-t_{o}\right)}{p_{\alpha}-1}\right)^{p_{\alpha}-1}\right)$ if $r_{\alpha}=1$. The exponents $r_{\alpha}$ and $p_{\alpha}$ have the following properties (see the proof of Lemma A.3 in $[12])$ :

$$
\begin{array}{ll}
\alpha^{*}(s x) \leq s^{\frac{p_{\alpha}}{p_{\alpha}-1}} \alpha^{*}(x), \quad \forall x \geq 0, \forall s \in[0,1] \\
\alpha^{*}(s x) \leq s^{\frac{r_{\alpha}}{r_{\alpha}-1}} \alpha^{*}(x), \quad \forall x \geq 0, \forall s>1 .
\end{array}
$$

Let $H(t)=\log \left\|e^{Q_{t} f}\right\|_{k(t)}$, with $f: X \rightarrow \mathbb{R}$ bounded and continuous. According to Proposition 4.1, we have, for all $t>0$,

$$
\frac{d H}{d t_{+}}(t) \leq \frac{k^{\prime}(t)}{k^{2}(t)} \frac{1}{\int e^{k(t) Q_{t} f} d \mu}\left[\operatorname{Ent}_{\mu}\left(e^{k(t) Q_{t} f}\right)+\frac{k^{2}(t)}{k^{\prime}(t)} \int \frac{d}{d t_{+}} Q_{t} f e^{k(t) Q_{t} f} d \mu\right] .
$$

The function $k(t) Q_{t} f$ belongs to $\mathcal{F}_{\alpha}$. Indeed, if $\ell=\lim _{x \rightarrow \infty} \alpha(x) / x=\infty$, this follows immediately from Lemma $4.2(2)$ below and the definition of $\mathcal{F}_{\alpha}$. On the other hand, if $\ell<+\infty$, then Lemma 4.2 (1) implies that $r_{\alpha}=1$, which in turn implies that $k(t) \leq 1$. By Lemma $4.2(2), Q_{t} f \in \mathcal{F}_{\alpha}$, which, in this case, means that $\operatorname{Lip}\left(Q_{t} f\right) \leq \ell$. Therefore, $\operatorname{Lip}\left(k(t) Q_{t} f\right) \leq \ell$ and so $k(t) Q_{t} f \in \mathcal{F}_{\alpha}$. Applying $\mathbf{L S I}_{\alpha}^{-}(C)$ to the function $k(t) Q_{t} f$, it follows that for all $t>0$ (or all $0<t \leq t_{o}$ if $r_{\alpha}=1$ ),

$$
\begin{aligned}
\operatorname{Ent}_{\mu}\left(e^{k(t) Q_{t} f}\right) & \leq C \int \alpha^{*}\left(k(t)\left|\nabla^{-} Q_{t} f\right|\right) e^{k(t) Q_{t} f} d \mu \\
& \leq C\left(k(t)^{\frac{p_{\alpha}}{p_{\alpha}-1}} \mathbf{1}_{t \leq t_{o}}+k(t)^{\frac{r_{\alpha}}{r_{\alpha}-1}} \mathbf{1}_{t>t_{o}}\right) \int \alpha^{*}\left(\left|\nabla^{-} Q_{t} f\right|\right) e^{k(t) Q_{t} f} d \mu \\
& \leq-C\left(k(t)^{\frac{p_{\alpha}}{p_{\alpha}-1}} \mathbf{1}_{t \leq t_{o}}+k(t)^{\frac{r_{\alpha}}{r_{\alpha}-1}} \mathbf{1}_{t>t_{o}}\right) \int \frac{d}{d t_{+}} Q_{t} f e^{k(t) Q_{t} f} d \mu
\end{aligned}
$$


where the last inequality follows from the Hamilton-Jacobi differential inequality (1.8). Therefore,

$$
\frac{d H}{d t_{+}}(t) \leq \frac{1-C k^{\prime}(t)\left(k(t)^{\frac{2-p_{\alpha}}{p_{\alpha}-1}} \mathbf{1}_{t \leq t_{o}}+k(t)^{\frac{2-r_{\alpha}}{r_{\alpha}-1}} \mathbf{1}_{t>t_{o}}\right)}{\int e^{k(t) Q_{t} f} d \mu} \int \frac{d}{d t_{+}} Q_{t} f e^{k(t) Q_{t} f} d \mu=0,
$$

where the last equality is a consequence of the very definition of $k$. Hence $H$ is non-increasing on $(0,+\infty)$ (or on $\left(0, t_{o}\right]$ if $r_{\alpha}=1$ ). When $\alpha(h) / h \rightarrow \infty$, when $h \rightarrow \infty$, then by point (3) of Proposition A.1 and the dominated convergence theorem, there holds

$$
\log \left\|e^{Q_{t} f}\right\|_{k(t)}=H(t) \leq \lim _{s \rightarrow 0^{+}} H(s)=\log \left\|e^{f}\right\|_{k(0)} .
$$

If $\alpha(h) / h \rightarrow \ell \in \mathbb{R}^{+}$, when $h \rightarrow \infty$, then by point (3) of Proposition A.1, the same conclusion holds if $\operatorname{Lip}(f)<\ell$. Consider now a bounded continuous function $f: X \rightarrow \mathbb{R}$ and fix $\varepsilon \in(0,1)$. Thanks to Lemma 4.2 below, $\operatorname{Lip}\left((1-\varepsilon) Q_{s} f\right)$ $\leq(1-\varepsilon) \ell$ for all $s>0$.

Since $Q_{s} f \leq f$, we conclude that

$$
\left\|e^{Q_{t}\left((1-\varepsilon) Q_{s} f\right)}\right\|_{k(t)} \leq\left\|e^{(1-\varepsilon) Q_{s} f}\right\|_{k(0)} \leq\left\|e^{(1-\varepsilon) f}\right\|_{k(0)} .
$$

Using Lebesgue's Theorem and Lemma 4.2, as $\varepsilon \rightarrow 0$, we get

$$
\left\|e^{Q_{t}\left(Q_{s} f\right)}\right\|_{k(t)} \leq\left\|e^{f}\right\|_{k(0)}
$$

Since $Q_{t+s} f \leq Q_{t}\left(Q_{s} f\right)$ and thanks to point (2) of Proposition A.1, we have $\lim _{s \rightarrow 0} Q_{t+s} f=Q_{t} f$ so that (using Lebesgue's theorem) the hypercontractivity property (4.1) still holds when $f$ is bounded and continuous, as expected.

Now we prove that if (4.1) holds for all bounded continuous $f$ and all $t>0$ with $k$ defined by (4.2), then $\mu$ satisfies $\mathbf{L S I}_{\alpha}^{-}(C)$. Observe that in the case $\alpha(h) / h \rightarrow \ell \in \mathbb{R}^{+}$, it is enough to show that $\mathbf{L S I}_{\alpha}^{-}$holds for functions with $\operatorname{Lip}(f)<\ell$.

Let $H(t)=\log \left\|e^{Q_{t} f}\right\|_{k(t)}$, for all $t>0$, with $f \in \mathcal{F}_{\alpha}$ and $\operatorname{Lip}(f)<\ell$ when $\alpha(h) / h \rightarrow \ell \in \mathbb{R}^{+}$as $h \rightarrow \infty$. By assumption, there holds

$$
\limsup _{t \rightarrow 0^{+}} \frac{H(t)-H\left(0^{+}\right)}{t} \leq 0 .
$$

We choose $t_{o}<C\left(p_{\alpha}-1\right)$ in the definition of $k(t)$ so that $k(0)$ and $k^{\prime}(0)>0$. It is not difficult to check that

$$
\begin{aligned}
& \limsup _{t \rightarrow 0^{+}} \frac{H(t)-H\left(0^{+}\right)}{t} \\
& \quad=\frac{k^{\prime}(0)}{k(0)^{2}} \frac{\operatorname{Ent}_{\mu}\left(e^{k(0) f}\right)}{\int e^{k(0) f} d \mu}-\frac{1}{k(0) \int e^{k(0) f} d \mu} \liminf _{t \rightarrow 0^{+}} \int \frac{e^{k(t) f}-e^{k(t) Q_{t} f}}{t} d \mu .
\end{aligned}
$$


According to the mean value theorem, there exists a function $\varphi:(0, \infty) \times X \rightarrow \mathbb{R}$ taking values in the interval $\left[k(t) e^{k(t) Q_{t} f(x)} ; k(t) e^{k(t) f(x)}\right]$ such that

$$
\frac{e^{k(t) f}-e^{k(t) Q_{t} f}}{t}=\frac{f-Q_{t} f}{t} \varphi(t, x), \quad \forall t>0, x \in X .
$$

Applying point (4) of Proposition A.1, we get

$$
\begin{aligned}
\liminf _{t \rightarrow 0^{+}} \int \frac{e^{k(t) f}-e^{k(t) Q_{t} f}}{t} d \mu & \leq \limsup _{t \rightarrow 0^{+}} \int \frac{e^{k(t) f}-e^{k(t) Q_{t} f}}{t} d \mu \\
& \leq k(0) \int \alpha^{*}\left(\left|\nabla^{-} f\right|\right) e^{k(0) f} d \mu
\end{aligned}
$$

Hence

$$
\operatorname{Ent}_{\mu}\left(e^{k(0) f}\right) \leq \frac{k(0)^{2}}{k^{\prime}(0)} \int \alpha^{*}\left(\left|\nabla^{-} f\right|\right) e^{k(0) f} d \mu
$$

Since

$$
k(0)=\left(1-\frac{C^{-1} t_{o}}{p_{\alpha}-1}\right)^{p_{\alpha}-1} \rightarrow 1 \quad \text { and } \quad k(0)^{2} / k^{\prime}(0)=C\left(1-\frac{C^{-1} t_{o}}{p_{\alpha}-1}\right)^{p_{\alpha}} \rightarrow C,
$$

when $t_{o} \rightarrow 0^{+}$, we conclude that $\mathbf{L S I}_{\alpha}^{-}(C)$ holds. This completes the proof.

In the proof above, we used the following technical lemma, whose proof is postponed to the appendix to preserve the continuity of the exposition.

Lemma 4.2. Set $\ell=\lim _{h \rightarrow \infty} \alpha(h) / h \in \mathbb{R} \cup\{+\infty\}$. Let $f: X \rightarrow \mathbb{R}$ be a bounded and continuous function. Then:

(1) If $\ell<+\infty$, then $r_{\alpha}=\inf _{x>0} x \alpha^{\prime}(x) / \alpha(x)=1$.

(2) For all $t>0, Q_{t} f \in \mathcal{F}_{\alpha}$.

(3) For all $t>0$ and all $x \in X, \lim _{\varepsilon \rightarrow 0} Q_{t}((1-\varepsilon) f)(x)=Q_{t} f(x)$.

We are now in position to derive the Otto-Villani theorem from Theorem 1.3.

Recall that, according to Bobkov and Götze's characterization, [5], $\mu$ satisfies the transport-entropy inequality $\mathbf{T}_{\alpha}(C)$ if and only if

$$
\int e^{C^{-1} Q_{1} f} d \mu \leq \exp \left(C^{-1} \int f d \mu\right)
$$

for all bounded continuous function $f: X \rightarrow \mathbb{R}$.

Proof of Theorem 1.4. Since $\mu$ satisfies $\mathbf{L S I}_{\alpha}^{-}(C)$, it satisfies the hypercontractivity property (1.9) of Theorem 1.3. Take $t_{o}=C\left(p_{\alpha}-1\right)$ in the definition of $k(t)$. The hypercontractivity inequality (1.9) yields

$$
\int e^{k(t) Q_{t} f} d \mu \leq e^{k(t) \int f d \mu}, \quad \forall t>0
$$


for all bounded continuous function $f$. By (4.3), this means that $\mu$ satisfies the following family of transport-entropy inequalities:

$$
\mathcal{T}_{\alpha(\cdot / t)}(\mu, \nu) \leq \frac{1}{t k(t)} H(\nu \mid \mu), \quad \forall \nu \in \mathcal{P}(X),
$$

where $\alpha(\cdot / t)$ denotes the function $x \mapsto \alpha(x / t)$. According to the proof of Lemma A.3 in $[12]$,

$$
\alpha(x) \leq \max \left(t^{r_{\alpha}} ; t^{p_{\alpha}}\right) \alpha(x / t), \quad \forall t>0 .
$$

Therefore, $\mu$ satisfies $\mathbf{T}_{\alpha}(A)$, with the constant

$$
A=\inf _{t>0} \frac{\max \left(t^{r_{\alpha}-1} ; t^{p_{\alpha}-1}\right)}{k(t)} .
$$

Taking $t=C\left(p_{\alpha}-1\right)$ for which $k(t)=1$, we see that

$$
A \leq \max \left(\left(\left(p_{\alpha}-1\right) C\right)^{r_{\alpha}-1} ;\left(\left(p_{\alpha}-1\right) C\right)^{p_{\alpha}-1}\right),
$$

which ends the proof.

Proof of Proposition 1.5. Define for all $t>0$ the operators

$$
R_{t} f(x)=\inf _{y \in X}\left\{f(y)+\frac{1}{t} \theta(d(x, y))\right\} \quad \text { and } \quad Q_{t} f(x)=\inf _{y \in X}\left\{f(y)+\frac{1}{t} d^{2}(x, y)\right\}
$$

According to Bobkov and Götze's dual formula (4.3) and by homogeneity, there holds, for all $t>0$,

$$
\int e^{C^{-1} t R_{t} f} d \mu \leq e^{C^{-1} t \int f d \mu}
$$

for all bounded continuous function $f$. Take a function $f$ such that $|f| \leq M$ and $\operatorname{Lip}(f, r)<\infty$ for some $r>0$. If $d(x, y) \geq a$, and $t \leq a^{2} /(2 M)$, then there holds

$$
f(y)+\frac{1}{t} \theta(d(x, y)) \geq-M+\frac{(2 M)}{a^{2}} a^{2}=M \geq f(x) \geq R_{t} f(x) .
$$

It follows that if $t \leq a^{2} / 2 M$, then

$$
\left.R_{t} f(x) \geq \inf _{y: d(x, y) \leq a}\left\{f(y)+\frac{1}{t} d^{2}(x, y)\right)\right\} \geq Q_{t} f(x) .
$$

Hence the following inequality holds:

$$
\int e^{C^{-1} t Q_{t} f} d \mu \leq e^{C^{-1} t \int f d \mu}, \quad \forall t \leq a^{2} /(2 M) .
$$

Applying Taylor's formula, we see that

$$
e^{C^{-1} t Q_{t} f(x)}=1+C^{-1} t Q_{t} f(x)+\frac{C^{-2}\left(t Q_{t} f\right)^{2}(x)}{2} e^{\varphi(t, x)},
$$


where $|\varphi(t, x)| \leq t C^{-1} M$, for all $t$ and $x$. So, for all $t \leq a^{2} /(2 M)$,

$$
\begin{aligned}
C^{-1} \int \frac{Q_{t} f-f}{t} d \mu+\frac{C^{-2}}{2} \int\left(Q_{t} f\right)^{2}(x) e^{\varphi(t, x)} & \mu(d x) \\
& \leq \frac{e^{C^{-1} t \int f d \mu}-1-t C^{-1} \int f d \mu}{t^{2}} .
\end{aligned}
$$

Letting $t$ go to 0 and using points (3) and (4) of Proposition A.1 together with the dominated convergence theorem yields

$$
-\frac{C^{-1}}{4} \int\left|\nabla^{-} f\right|^{2} d \mu+\frac{C^{-2}}{2} \int f^{2} d \mu \leq \frac{C^{-2}}{2}\left(\int f d \mu\right)^{2},
$$

which is the claimed Poincaré inequality.

\section{Transport-entropy inequalities as restricted log-Sobolev inequalities}

In this section, we show that a transport-entropy inequality can be characterized as a modified log-Sobolev inequality restricted to a class of $c$-convex functions. Actually we will prove the following improved version of Theorem 1.6 which holds even if the space is not geodesic.

Theorem 5.1. Let $\mu$ be a probability measure on $(X, d)$ and let $p \geq 2$. Define the function $\beta_{p}$ by

$$
\beta_{p}(u)=\frac{u}{\left[u^{1 /(p-1)}-1\right]^{p-1}}, \quad \forall u>1 .
$$

The following properties are equivalent:

(1) There is some $C>0$ such that $\mu$ satisfies $\mathbf{T}_{p}(C)$.

(2) There is some $D>0$ such that $\mu$ satisfies the following $(\tau)$-log-Sobolev inequality: for all bounded continuous $f$ and all $0<\lambda<1 / D$, there holds

$$
\operatorname{Ent}_{\mu}\left(e^{f}\right) \leq \frac{1}{1-\lambda D} \int\left(f-Q^{\lambda} f\right) e^{f} d \mu,
$$

where for all $\lambda>0, Q^{\lambda} f(x)=\inf _{y \in X}\left\{f(y)+\lambda c_{p}(x, y)\right\}$.

(3) There is some $E>0$ such that $\mu$ satisfies the following restricted log-Sobolev inequality: for all $K c_{p}$-convex function $f$, with $0<K<1 / E$ there holds

$$
\operatorname{Ent}_{\mu}\left(e^{f}\right) \leq \frac{\beta_{p}(u)-1}{(1-K E u) p K^{q-1}} \int\left|\nabla_{K c_{p}}^{-} f\right|^{q} e^{f} d \mu, \quad \forall u \in(1,1 /(K E))
$$

where $q=p /(p-1)$ and $\left|\nabla_{K c_{p}}^{-} f\right|(x)=K\left(\inf _{\bar{y} \in \partial_{K c_{p}} f(x)} d(x, \bar{y})\right)^{p-1}$ (see Proposition 2.9).

Moreover, when the space $(X, d)$ is geodesic these properties are equivalent to the following: 
(3') There is some $F>0$ such that $\mu$ satisfies the following restricted log-Sobolev inequality: for all $K c_{p}$-convex function $f$, with $0<K<1 / F$, there holds

$$
\operatorname{Ent}_{\mu}\left(e^{f}\right) \leq \frac{\beta_{p}(u)-1}{(1-K F u) p K^{q-1}} \int\left|\nabla^{+} f\right|^{q} e^{f} d \mu, \quad \forall u \in(1,1 /(K F))
$$

The optimal constants $C_{\mathrm{opt}}, D_{\mathrm{opt}}, E_{\mathrm{opt}}$ and $F_{\mathrm{opt}}$ are related by

$$
F_{\text {opt }} \leq E_{\text {opt }} \leq D_{\text {opt }} \leq C_{\text {opt }} \leq \kappa_{p} F_{\text {opt }},
$$

where $\kappa_{p}$ is some universal constant depending only on $p$. For $p=2$, one can take $\kappa_{2}=e^{2}$.

\subsection{From transport-entropy inequalities to $(\tau)$-log-Sobolev inequalities}

We recall the following proposition from [11], whose proof relies on a simple Jensen argument.

Lemma 5.2. If $\mu$ satisfies the transport-entropy property $\mathbf{T}_{c}(C)$, for some continuous cost function $c$ on $X^{2}$, then the following $(\tau)$-log-Sobolev property holds: for all function $f$, for all $0<\lambda<1 / C$,

$$
\operatorname{Ent}_{\mu}\left(e^{f}\right) \leq \frac{1}{1-\lambda C} \int\left(f-Q^{\lambda} f\right) e^{f} d \mu
$$

where for all $x \in X, Q^{\lambda} f(x)=\inf \{f(y)+\lambda c(x, y)\}$.

This proves the step $(1) \Rightarrow(2)$ in Theorem 5.1 .

\subsection{From $(\tau)$-log-Sobolev inequalities to log-Sobolev inequalities for $c_{p}$-convex functions}

The general link between the $(\tau)$-log-Sobolev property and the restricted logSobolev inequality is the following: if the function $f$ is $c$-convex then the quantity $f-Q^{\lambda} f$ on the right-hand side of $(5.2)$ can be bounded by a function of $\left|\nabla_{c}^{-} f\right|$ (see Lemma 5.3 below).

From now on, we assume that $c=c_{p}$ is the cost function defined by: for all $x, y$ in $X, c_{p}(x, y)=d^{p}(x, y) / p$, for some $p>1$.

Lemma 5.3. Let $\lambda>0$. If $f$ is a $K c_{p}$-convex function bounded from above, and if $0<K<\lambda$, then for all $x \in X$ and all $\bar{y}$ in the $K c_{p}$-subdifferential $\partial_{K c_{p}} f(x)$ of $f$ at point $x$,

$$
f(x)-Q^{\lambda} f(x) \leq K\left(\beta_{p}(\lambda / K)-1\right) c_{p}(x, \bar{y}),
$$

where $Q^{\lambda} f(x)=\inf _{y \in X}\left\{f(y)+\lambda c_{p}(x, y)\right\}$ and for all $u>1, \beta_{p}(u)=\frac{u}{\left[u^{1 /(p-1)}-1\right]^{p-1}}$.

Equivalently, with the notation of Proposition 2.9,

$$
f(x)-Q^{\lambda} f(x) \leq\left(\beta_{p}(\lambda / K)-1\right) \frac{1}{p K^{q-1}}\left|\nabla_{K c_{p}}^{-} f\right|^{q}(x),
$$

where $q=p /(p-1)$. 
Proof. By the definition (Definition 2.4) of $\partial_{K c_{p}} f(x)$ and using the triangular inequality we get, for all $\bar{y} \in \partial_{K c_{p}} f(x)$,

$$
\begin{aligned}
f(x) & -Q^{\lambda} f(x)=\sup _{z \in X}\left\{f(x)-f(z)-\lambda c_{p}(z, x)\right\} \\
& \leq \sup _{z \in X}\left\{K c_{p}(z, \bar{y})-K c_{p}(x, \bar{y})-\lambda c_{p}(z, x)\right\} \\
& \leq \sup _{z \in X}\left\{K c_{p}(z, \bar{y})-\lambda c_{p}(z, x)\right\}-K c_{p}(x, \bar{y}) \\
& \leq \frac{1}{p} \sup _{z \in X}\left\{K(d(z, x)+d(x, \bar{y}))^{p}-\lambda d^{p}(z, x)\right\}-K c_{p}(x, \bar{y}) \\
& \leq \frac{1}{p} \sup _{r \geq 0}\left\{K(r+d(x, \bar{y}))^{p}-\lambda r^{p}\right\}-K c_{p}(x, \bar{y})=K c_{p}(x, \bar{y})\left(\beta_{p}(\lambda / K)-1\right) .
\end{aligned}
$$

Thus optimizing over all possible $\bar{y} \in \partial_{K c_{p}} f(x)$ yields the expected result

$$
\begin{aligned}
f(x)-Q^{\lambda} f(x) & \leq\left(\beta_{p}(\lambda / K)-1\right) \inf _{\bar{y} \in \partial_{K c_{p}} f(x)} K c_{p}(x, \bar{y}) \\
& =\left(\beta_{p}(\lambda / K)-1\right) \frac{1}{p K^{q-1}}\left|\nabla_{K c_{p}}^{-} f\right|^{q}(x) .
\end{aligned}
$$

From this lemma the $(\tau)$-log-Sobolev property (5.2) provides immediately the first part of the following statement by setting $u=\lambda / C$.

Proposition 5.4. If $\mu$ satisfies the $(\tau)$-log-Sobolev (5.2) with the cost $c=c_{p}$, $p \geq 2$, then for all $K \in(0,1 / C)$ and all functions $f$ that are bounded from above and $K c_{p}$-convex, there holds

$$
\operatorname{Ent}_{\mu}\left(e^{f}\right) \leq \frac{\beta_{p}(u)-1}{(1-K C u) p K^{q-1}} \int\left|\nabla_{K c_{p}}^{-} f\right|^{q}(x) e^{f(x)} \mu(d x), \quad \forall u \in(1,1 /(K C)) .
$$

Moreover, when $(X, d)$ is geodesic, the same inequality holds with $\left|\nabla^{+} f\right|$ instead of $\left|\nabla_{K_{c_{p}}}^{-} f\right|$ on the right-hand side.

This proves the implications $(2) \Rightarrow(3)$ and $(2) \Rightarrow\left(3^{\prime}\right)$ (in the geodesic case) in Theorem 5.1.

Proof. Let us justify the statement in the geodesic case. According to Proposition 2.9 (applied with the function $\theta(x)=K x^{p} / p$ ), there holds $\left|\nabla_{K c_{p}}^{-} f\right| \leq\left|\nabla_{K c_{p}}^{+} f\right|$ and when the space is geodesic, $\left|\nabla_{K c_{p}}^{+} f\right|=\left|\nabla^{+} f\right|$, which completes the proof.

\subsection{From log-Sobolev inequalities for $c_{p}$-convex functions to transport- entropy inequalities}

In this part we prove that a modified log-Sobolev inequality restricted to the class of $K c_{p}$-convex functions also implies a transport entropy-inequality. One of the main ingredient of the proof is Theorem 1.2. 
Theorem 5.5. Let $p \geq 2$. Suppose that for all $K \in(0,1 / C)$ and all $K c_{p}$-convex functions $f: X \rightarrow \mathbb{R}$ bounded from above, there holds

$$
\operatorname{Ent}_{\mu}\left(e^{f}\right) \leq \frac{\beta_{p}(u)-1}{(1-K C u) p K^{q-1}} \int\left|\nabla_{K c_{p}}^{-} f\right|^{q}(x) e^{f(x)} \mu(d x), \quad \forall u \in\left(1, \frac{1}{K C}\right) .
$$

Then $\mu$ satisfies the inequality $\mathbf{T}_{p}\left(\kappa_{p} C\right)$, where $\kappa_{p}$ is some numerical constant depending only on $p$. For $p=2, \kappa_{2}=e^{2}$. Moreover, if the space is geodesic, the same conclusion holds if $\left|\nabla_{K c_{p}}^{-} f\right|$ is replaced by $\left|\nabla^{+} f\right|$ on the right-hand side of (5.3).

This proves the implications $(3) \Rightarrow(1)$ and $\left(3^{\prime}\right) \Rightarrow(1)$ (in the geodesic case) and completes the proof of Theorem 5.1.

Proof. For any bounded continuous function $g$, we define the function $P_{t} g$ by

$$
P_{t} g(x)=\sup _{y \in X}\left\{g(y)-\frac{1}{t^{p-1}} c_{p}(x, y)\right\} .
$$

Let $\ell:[a, 1] \rightarrow(0,+\infty)$ be a decreasing $\mathcal{C}^{1}$ function defined on some interval $[a, 1]$ with $a>0$ and such that $\ell(1)=0$. For all bounded continuous $g$ define

$$
H_{g}(t)=\frac{C}{\ell(t)} \log \left(\int e^{C^{-1} \ell(t) P_{t} g} d \mu\right), \quad t \in[a, 1) .
$$

If all the $H_{g}$ 's were non-decreasing, then it would hold that $H_{g}(a) \leq \lim _{t \rightarrow 1^{-}} H_{g}(t)=$ $\int P_{1} g d \mu$. Since $g \leq P_{a} g$, we would get

$$
\int e^{C^{-1} \ell(a) g} d \mu \leq e^{C^{-1} \ell(a) \int P_{1} g d \mu}
$$

which in turn, according to Bobkov and Götze characterization theorem, would prove that $\mu$ satisfies $\mathbf{T}_{p}(C / \ell(a))$.

Hence, our aim is to construct a function $\ell$ such that all the $H_{g}$ 's are nondecreasing. Set $f_{t}=C^{-1} \ell(t) P_{t} g$. According to Proposition $4.1, H_{g}$ is continuous and differentiable on the right and

$$
\frac{d}{d t_{+}} H_{g}(t)=\frac{C \ell^{\prime}(t)}{\ell^{2}(t) \int e^{f_{t}} d \mu}\left[\operatorname{Ent}_{\mu}\left(e^{f_{t}}\right)+\frac{\ell(t)^{2}}{C \ell^{\prime}(t)} \int \frac{d P_{t} g}{d t_{+}} e^{f_{t}} d \mu\right] .
$$

Since $\ell^{\prime}<0$, all we have to show is that the term into brackets is non-positive. For all $t>0$, the function $f_{t}$ is $K(t) c_{p^{-}}$convex, with $K(t)=\frac{\ell(t)}{C t^{p-1}}$. Hence, for all $t$ such that $\ell(t)<t^{p-1}$ and all $u \in(1,1 /(C K(t))$,

$$
\operatorname{Ent}_{\mu}\left(e^{f_{t}}\right) \leq \frac{\beta_{p}(u)-1}{(1-K(t) C u) p K(t)^{q-1}} \int\left|\nabla_{K(t) c_{p}}^{-}\left(f_{t}\right)\right|^{q}(x) e^{f_{t}(x)} \mu(d x) .
$$

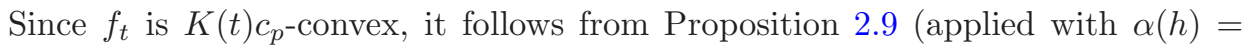
$\left.K(t) h^{p} / p\right)$ that

$$
\left|\nabla_{K(t) c_{p}}^{-} f_{t}\right|(x)=K(t)\left(\min _{\bar{y} \in \partial_{K(t) c_{p}} f_{t}(x)} d(x, \bar{y})\right)^{p-1} \leq K(t)\left(\max _{\bar{y} \in m(t, x)} d(x, \bar{y})\right)^{p-1}
$$


where $m(t, x)$ is the set of points $\bar{y}$ where the supremum defining $P_{t} g$ is attained. As a result, there holds

$$
\frac{1}{p K(t)^{q-1}}\left|\nabla_{K(t) c_{p}}^{-} f_{t}\right|^{q}(x) \leq K(t) \max _{\bar{y} \in m(t, x)} c_{p}(x, \bar{y}) .
$$

On the other hand, according to Proposition 1.2,

$$
\frac{d P_{t} g}{d t_{+}}(x)=\frac{p-1}{t^{p}} \max _{\bar{y} \in m(t, x)} c_{p}(x, \bar{y}) .
$$

Therefore

$$
\frac{1}{p K(t)^{q-1}}\left|\nabla_{K(t) c_{p}}^{-} f_{t}\right|^{q}(x) \leq \frac{K(t) t^{p}}{(p-1)} \frac{d P_{t} g}{d t_{+}}(x)=\frac{t \ell(t)}{(p-1) C} \frac{d P_{t} g}{d t_{+}}(x) .
$$

So, for all $t>0$ with $\ell(t)<t^{p-1}$, there holds

$$
\begin{aligned}
& {\left[\operatorname{Ent}_{\mu}\left(e^{f_{t}}\right)+\frac{\ell(t)^{2}}{C \ell^{\prime}(t)} \int \frac{d P_{t} g}{d t_{+}} e^{f_{t}} d \mu\right]} \\
& \leq \frac{\ell(t)}{C}\left[\theta_{p}\left(\frac{\ell(t)}{t^{p-1}}\right) \frac{t}{p-1}+\frac{\ell(t)}{\ell^{\prime}(t)}\right] \int \frac{d P_{t} g}{d t_{+}} e^{f_{t}} d \mu
\end{aligned}
$$

where the function $\theta_{p}$ is defined by $\theta_{p}(x)=\inf _{1<u<1 / x}\left\{\frac{\beta_{p}(u)-1}{1-x u}\right\}$, for $x<1$. Observe that $\theta_{p}$ is finite on $[0,1[$. Consider the function

$$
\Psi_{p}(r)=\frac{1}{p-1} \int_{0}^{r} \frac{\theta_{p}(s)}{s\left(\theta_{p}(s)+1\right)} d s, \quad \forall r \in[0,1] .
$$

According to Lemma 5.6 below, since $p \geq 2$, the function $\Psi_{p}$ is well defined, increasing and of class $\mathcal{C}^{1}$ on $(0,1)$. Define $v(t)=\Psi_{p}^{-1}(-\ln (t))$, for all $t \in\left[a_{p}, 1\right]$, with $a_{p}=\exp \left(-\Psi_{p}(1)\right)$. The function $v$ is decreasing and $v(t) \in[0,1]$ for all $t \in\left[a_{p}, 1\right]$. Finally, define $\ell_{p}(t)=t^{p-1} v(t)$, for all $t \in\left[a_{p}, 1\right]$. A simple calculation shows that

$$
\theta_{p}\left(\frac{\ell_{p}(t)}{t^{p-1}}\right) \frac{t}{p-1}+\frac{\ell_{p}(t)}{\ell_{p}^{\prime}(t)}=0, \quad \forall t \in\left(a_{p}, 1\right) .
$$

We conclude that $\mu$ satisfies the inequality $\mathbf{T}_{p}$ with the constant

$$
\frac{C}{\ell_{p}\left(a_{p}\right)}=C \exp \left(\int_{0}^{1} \frac{\theta_{p}(s)}{s\left(\theta_{p}(s)+1\right)} d s\right)=C \kappa_{p} .
$$

In the particular case $p=2$, one has $\theta_{2}(x)=4 x /(1-x)^{2}$, and it is easy to check that $\kappa_{2}=e^{2}$.

There remains to consider the geodesic case. In this case, the inequality (5.4) is replaced by the equality

$$
\frac{1}{p K(t)^{q-1}}\left|\nabla^{+} f_{t}\right|^{q}(x)=\frac{K(t) t^{p}}{(p-1)} \frac{d P_{t} g}{d t_{+}}(x),
$$

and the rest of the proof remains unchanged. 
Lemma 5.6. The function $s \mapsto \phi(s)=\frac{\theta_{p}(s)}{s\left(\theta_{p}(s)+1\right)}$ is continuous on $(0,1)$. Moreover, $\phi(s)$ goes to 1 as $s$ goes to 1 , and

$$
\phi(s)=\frac{p^{p /(p-1)}}{s^{(p-2) /(p-1)}}(1+\varepsilon(s)), \quad \text { with } \varepsilon(s) \rightarrow 0 \text { as } s \rightarrow 0 .
$$

Proof. After some computations, it is easy to check that for $s \in(0,1)$, the infimum $\theta_{p}(s)$ is attained at some unique point $u=u(s) \in(1,1 / s)$ such that

$$
\beta_{p}^{\prime}(u)(1-s u)+s\left(\beta_{p}(u)-1\right)=0,
$$

or equivalently

$$
u(s)^{p /(p-1)}-\left(u(s)^{1 /(p-1)}-1\right)^{p}=1 / s .
$$

It follows from this equality that $u(s)$ is continuous on $(0,1), u(s) \rightarrow 1$ as $s \rightarrow 1$ and $u(s) \rightarrow+\infty$ as $s \rightarrow 0$. As a first consequence, $\phi$ is continuous on $(0,1)$.

By a Taylor expansion at 0 , one has

$$
\frac{1}{s u(s)^{p /(p-1)}}=1-\left(1-\frac{1}{u(s)^{1 /(p-1)}}\right)^{p}=\frac{p}{u(s)^{1 /(p-1)}}(1+\varepsilon(s)),
$$

with $\varepsilon(s) \rightarrow 0$ as $s \rightarrow 0$. It follows that $s u(s) \rightarrow 1 / p$ as $s \rightarrow 0$. From these observations, we get

$$
\phi(s)=\frac{1-\left(1-u(s)^{-1 /(p-1)}\right)^{p-1}}{s\left(1-s u(s)\left(1-u(s)^{-1 /(p-1)}\right)^{p-1}\right)}=\frac{p^{p /(p-1)}}{s^{(p-2) /(p-1)}}(1+\varepsilon(s)),
$$

with $\varepsilon(s) \rightarrow 0$ as $s \rightarrow 0$. Since $u(s) \rightarrow 1$ as $s \rightarrow 1$ we easily get that $\phi(s) \rightarrow 1$ as $s \rightarrow 1$.

\section{A. Proofs of Lemma 2.8, Lemma 2.11, Proposition 4.1 and Lemma 4.2}

In this appendix we give the technical proofs of Lemmas 2.8, 2.11 and 4.2, and of Proposition 4.1.

Proof of Lemma 2.8. Let $\bar{y} \in \partial_{c} f(x)$. According to the definition of the $c$-subdifferential,

$$
f(z)-f(x) \geq L(x-\bar{y})-L(z-\bar{y}), \quad \forall z \in \mathbb{R}^{m} .
$$

Let $z=x+\varepsilon u$ with $\varepsilon>0$ and $u \in \mathbb{R}^{m}$. Since $L$ and $f$ are smooth at $x$, we get that, as $\varepsilon$ tends to 0 , for all $u \in \mathbb{R}^{m}$,

$$
u \cdot \nabla f(x) \geq-u \cdot \nabla L(x-\bar{y})
$$

and therefore $\nabla f(x)=-\nabla L(x-\bar{y})$. Let $v_{o}=x-\bar{y}$ and $u_{o}=\nabla L\left(v_{0}\right)$. By the convexity property of $L$,

$$
L(v) \geq L\left(v_{o}\right)+u_{o} \cdot\left(v-v_{o}\right), \quad \forall v \in \mathbb{R}^{m},
$$


or equivalently $L\left(v_{o}\right) \leq u_{o} \cdot v_{o}-L^{*}\left(u_{o}\right)$. Since $L\left(v_{o}\right)=\sup _{u \in \mathbb{R}^{m}}\left\{u \cdot v_{o}-L^{*}(u)\right\}$, it follows that the derivative of $u \mapsto u \cdot v_{o}-L^{*}(u)$ vanishes at $u_{o}$, and so $v_{o}=\nabla L^{*}\left(u_{o}\right)$. Finally, $x-\bar{y}=\nabla L^{*}\left(u_{o}\right)=\nabla L^{*}(-\nabla f(x))$, which completes the proof.

Proof of Lemma 2.11. (1) The function $h: y \mapsto g(y)-t \alpha(d(x, y) / t)$ is upper semicontinuous, bounded from above and its level sets $\{h \geq r\} r \in \mathbb{R}$ are compact. It follows that $h$ attains its supremum and so $m(t, x)=\{h \geq \sup h\}$ is not empty and compact.

(2) Let $h_{n}(y)=g(y)-t_{n} \alpha\left(d\left(x_{n}, y\right) / t_{n}\right), y \in X$. The sequence of functions $h_{n}$ converges pointwise to the function $h$, and the convergence is uniform on each bounded set. Since $g$ is bounded from above by some constant $r \in \mathbb{R}$, there holds

$$
\begin{aligned}
r-t_{n} \alpha\left(\frac{d\left(x_{n}, y_{n}\right)}{t_{n}}\right) & \geq g\left(y_{n}\right)-t_{n} \alpha\left(\frac{d\left(x_{n}, y_{n}\right)}{t_{n}}\right) \\
& \geq g(y)-t_{n} \alpha\left(\frac{d\left(x_{n}, y\right)}{t_{n}}\right), \quad \forall y \in X .
\end{aligned}
$$

Since $\left(x_{n}\right)_{n \in \mathbb{N}}$ is bounded and $\lim _{n \rightarrow \infty} t_{n}=t>0$, we conclude that $\left(y_{n}\right)_{n \in \mathbb{N}}$ is a bounded sequence. As balls are supposed to be compact, $\left(y_{n}\right)_{n \in \mathbb{N}}$ has converging subsequences. Passing to the limit in the inequality (A.2) along a converging subsequence of $\left(y_{n}\right)_{n \in \mathbb{N}}$ yields the conclusion that any limit point $\bar{y}$ of $\left(y_{n}\right)_{n \in \mathbb{N}}$ belongs to $m(t, x)$.

We turn to the proof of Proposition 4.1. The proof requires some regularity properties of $Q_{t} f$ in the $t$ variable that are gathered in the following proposition.

Proposition A.1. Let $f$ be a bounded lower semicontinuous function on $X$; define, for all $t>0$ and $x \in X$,

$$
Q_{t} f(x)=\inf \left\{f(y)+t \alpha\left(\frac{d(x, y)}{t}\right)\right\},
$$

and let $m(t, x)$ denote the set of points where this infimum is attained. The following properties hold:

(1) For all $x \in X$,

$$
m(t, x) \subset B\left(x, t \alpha^{-1}(\operatorname{Osc}(f) / t)\right)
$$

(2) For all $t, h>0$,

$$
\frac{1}{h} \sup _{x \in X}\left|Q_{t+h} f(x)-Q_{t} f(x)\right| \leq \beta\left(\alpha^{-1}(\operatorname{Osc}(f) / t)\right) .
$$

(3) If $\alpha(h) / h \rightarrow \infty$, when $h \rightarrow \infty$, then for all bounded continuous functions $f$ and for all $x \in X$,

$$
\lim _{t \rightarrow 0^{+}} Q_{t} f(x)=f(x)
$$

and

$$
\liminf _{t \rightarrow 0^{+}} \frac{Q_{t} f(x)-f(x)}{t} \geq-\alpha^{*}\left(\left|\nabla^{-} f\right|(x)\right) .
$$

If $\alpha(h) / h \rightarrow \ell \in \mathbb{R}^{+}$, when $h \rightarrow \infty$, the same conclusions hold for all functions $f$ with $\operatorname{Lip}(f)<\ell$. 
(4) Let $\mu$ be a probability measure and let $\varphi:(0,+\infty) \times X \rightarrow \mathbb{R}$ be such that $|\varphi| \leq M$ for some $M>0$ and $\lim _{t \rightarrow 0+} \varphi(t, x)=\psi(x)$ for all $x \in X$. If $\alpha(h) / h \rightarrow \infty$ when $h \rightarrow \infty$ and if $f$ is such that $\operatorname{Lip}(f, r)<+\infty$ for some $r>0$, then

$$
\limsup _{t \rightarrow 0} \int \frac{f-Q_{t} f}{t} \varphi(t, x) d \mu \leq \int \alpha^{*}\left(\left|\nabla^{-} f\right|(x)\right) \psi(x) d \mu .
$$

The same conclusion holds if $\alpha(h) / h \rightarrow \ell \in \mathbb{R}^{+}$when $h \rightarrow \infty$ and $\operatorname{Lip}(f)<\ell$.

Proof of Proposition A.1. (1) Let $M=\sup (f)$ and $m=\inf (f)$. If $\bar{y} \in m(t, x)$, there holds

$$
m+t \alpha\left(\frac{d(x, \bar{y})}{t}\right) \leq f(\bar{y})+t \alpha\left(\frac{d(x, \bar{y})}{t}\right)=Q_{t} f(x) \leq M,
$$

which proves the first claim.

(2) Since $t \mapsto Q_{t} f(x)$ is non-increasing, $\left|Q_{t+h} f(x)-Q_{t} f(x)\right|=Q_{t} f(x)-$ $Q_{t+h} f(x)$. If $\bar{y} \in m(t+h, x)$, then

$$
\begin{aligned}
\frac{1}{h}\left(Q_{t} f(x)-Q_{t+h} f(x)\right) & \leq \frac{1}{h}\left(t \alpha\left(\frac{d(x, \bar{y})}{t}\right)-(t+h) \alpha\left(\frac{d(x, \bar{y})}{t+h}\right)\right) \\
& \leq \beta\left(\alpha^{-1}(\operatorname{Osc}(f) / t)\right),
\end{aligned}
$$

where the last inequality comes from the mean value theorem, the monotonicity of the function $\beta$, and point (1).

(3) Let us first assume that $\alpha(h) / h \rightarrow+\infty$, when $h \rightarrow+\infty$. In this case, $t \alpha^{-1}(\mathrm{Osc}(f) / t) \rightarrow 0$ when $t \rightarrow 0$, and so, according to (1),

$$
\inf _{y \in B\left(x, t \alpha^{-1}(\operatorname{Osc}(f) / t)\right)}\{f(y)\} \leq Q_{t} f(x) \leq f(x) .
$$

Since $f$ is lower semicontinuous, the limit when $t$ goes to 0 of the left-hand side is greater than or equal to $f(x)$. This guarantees that $\lim _{t \rightarrow 0^{+}} Q_{t} f(x)=f(x)$. Moreover, for all $\bar{y}_{t} \in m(t, x), f\left(\bar{y}_{t}\right) \leq f(x)$ and therefore

$$
\begin{aligned}
\frac{f(x)-Q_{t} f(x)}{t} & =\frac{f(x)-f\left(\bar{y}_{t}\right)}{t}-\alpha\left(\frac{d\left(x, \bar{y}_{t}\right)}{t}\right) \\
& =\frac{\left[f\left(\bar{y}_{t}\right)-f(x)\right]_{-}}{d\left(x, \bar{y}_{t}\right)} \frac{d\left(x, \bar{y}_{t}\right)}{t}-\alpha\left(\frac{d\left(x, \bar{y}_{t}\right)}{t}\right) \leq \alpha^{*}\left(\frac{\left[f\left(\bar{y}_{t}\right)-f(x)\right]_{-}}{d\left(x, \bar{y}_{t}\right)}\right) .
\end{aligned}
$$

Arguing as before, we see that $\bar{y}_{t} \rightarrow x$ as $t \rightarrow 0$ so that

$$
\limsup _{t \rightarrow 0^{+}} \frac{f(x)-Q_{t} f(x)}{t} \leq \alpha^{*}\left(\left|\nabla^{-} f\right|(x)\right) .
$$

Now we assume that $\alpha(h) / h \rightarrow \ell \in \mathbb{R}^{+}$when $h \rightarrow \infty$. According to what precedes, it is enough to show that there is a constant $r>0$ such that

$$
m(t, x) \subset B(x ; r t), \quad \forall t>0, x \in X .
$$


Let $\bar{y} \in m(t, x)$. Then there holds $f(\bar{y})-f(x)+t \alpha(d(x, \bar{y}) / t) \leq 0$. Since $f$ is assumed to be Lipschitz, we conclude that $\operatorname{Lip}(f) d(x, \bar{y}) / t \geq \alpha(d(x, \bar{y}) / t)$. Since $\operatorname{Lip}(f)<\ell=\lim _{h \rightarrow+\infty} \alpha(h) / h$, this implies that $d(x, \bar{y}) \leq r t$ where $r=\sup \{h$ : $\alpha(h) / h \leq \operatorname{Lip}(f)\}<+\infty$, which proves the claim.

(4) We already know, by point (3), that

$$
\limsup _{t \rightarrow 0^{+}} \frac{f(x)-Q_{t} f(x)}{t} \leq \alpha^{*}\left(\left|\nabla^{-} f\right|(x)\right) .
$$

Hence the result of point (4) will follow from Fatou's Lemma (in its limsup version) as soon as for some $t_{0}>0$, there holds

$$
\sup _{x} \sup _{t \in\left(0, t_{o}\right)} \frac{f(x)-Q_{t} f(x)}{t}<\infty .
$$

Assume first that $\lim _{h \rightarrow \infty} \alpha(h) / h=\infty$ and let $r>0$ be such that $\operatorname{Lip}(f, r)<\infty$. Observe that $\lim _{t \rightarrow 0} t \alpha^{-1}(\operatorname{Osc}(f) / t)=0$ so that, by point $(1)$, there exists $t_{o}>0$ such that, for all $t \in\left(0, t_{o}\right)$, all $x \in X$ and all $\bar{y}_{t} \in m(t, x), d\left(x, \bar{y}_{t}\right) \leq r$. Using (A.3), we conclude that

$$
\sup _{x} \sup _{t \in\left(0, t_{o}\right)} \frac{f(x)-Q_{t} f(x)}{t} \leq \alpha^{*}(\operatorname{Lip}(f, r))<\infty .
$$

Assume now that $\alpha(h) / h \rightarrow \ell \in \mathbb{R}^{+}$, when $h \rightarrow \infty$. Then, since $\operatorname{Lip}(f)<\ell$, (A.3) implies that

$$
\sup _{x, t} \frac{f(x)-Q_{t} f(x)}{t} \leq \alpha^{*}(\operatorname{Lip}(f))<\infty .
$$

This ends the proof of point (4) and of the proposition.

Proof of Proposition 4.1. We will prove that $H$ is right differentiable, the proof of the left-differentiability being similar. Formally differentiating under the integral sign yields, for all $t>0$,

$$
\begin{aligned}
\frac{d H}{d t_{+}}(t)= & -\frac{k^{\prime}(t)}{k(t)^{2}} \log \left(\int e^{k(t) Q_{t} f} d \mu\right) \\
(\mathrm{A} .4) & +\frac{1}{k(t) \int e^{k(t) Q_{t} f} d \mu}\left[\int k^{\prime}(t) Q_{t} f e^{k(t) Q_{t} f} d \mu+\int k(t) \frac{d}{d t_{+}} Q_{t} f e^{k(t) Q_{t} f} d \mu\right]
\end{aligned}
$$

which easily gives the desired identity. Hence, there remains to justify the above calculation. Define $F(t)=\int e^{k(t) Q_{t} f} d \mu$. To obtain (A.4), it is enough to show that $F$ is right differentiable and that

$$
\frac{d F}{d t_{+}}(t)=\int k^{\prime}(t) Q_{t} f e^{k(t) Q_{t} f} d \mu+\int k(t) \frac{d}{d t_{+}} Q_{t} f e^{k(t) Q_{t} f} d \mu .
$$


For all $s>0, \frac{1}{s}(F(t+s)-F(t))=\int G_{s} d \mu$, with $G_{s}=\frac{1}{s}\left(e^{k(t+s) Q_{t+s} f}-e^{k(t) Q_{t} f}\right)$. Since $t \mapsto Q_{t} f(x)$ is right differentiable for $t>0$,

$$
G_{s}(x) \underset{s \rightarrow 0}{\longrightarrow} k^{\prime}(t) Q_{t} f(x) e^{k(t) Q_{t} f(x)}+k(t) \frac{d}{d t_{+}} Q_{t} f(x) e^{k(t) Q_{t} f(x)} .
$$

For a given $t \in(a, b)$, let $\eta_{t}>0$ be any number such that $t+\eta_{t}<b$. Then, using the mean value theorem together with point (2) of Proposition A.1, it is not difficult to prove that $\sup _{x \in X} \sup _{s \leq \eta_{t}}\left|G_{s}\right|(x)<+\infty$. Applying the dominated convergence theorem completes the proof.

Proof of Lemma 4.2. Let $f: X \rightarrow \mathbb{R}$ be a bounded and continuous function and fix $t>0$.

(2) First, following Lemma 3.8 in [12], we will prove that there exists $r>0$ such that $\operatorname{Lip}\left(Q_{t} f, r\right)<\infty$. Set $r=t \alpha^{-1}(\operatorname{Osc}(f) / t)$. By point (1) of Proposition A.1, there holds

$$
Q_{t} f(u)=\inf _{d(y, u) \leq r}\{f(y)+t \alpha(d(u, y) / t)\}, \quad \forall u \in X .
$$

Fix $u, v \in X$ with $d(u, v) \leq r$. Then, given $y_{o} \in X$ such that $d\left(v, y_{o}\right) \leq r$, it follows from the mean value theorem that

$$
\begin{aligned}
\mid t \alpha\left(d\left(u, y_{o}\right) / t\right) & -t \alpha\left(d\left(v, y_{o}\right) / t\right) \mid \\
& \leq\left|d\left(v, y_{o}\right)-d\left(u, y_{o}\right)\right| \max _{s \in[0,1]} \alpha^{\prime}\left(\left[s d\left(u, y_{o}\right)+(1-s) d\left(v, y_{o}\right)\right] / t\right) \\
& \leq \alpha^{\prime}(2 r / t) d(u, v) .
\end{aligned}
$$

Now, let $y_{o}$ be such that $Q_{t} f(v)=f\left(y_{o}\right)+t \alpha\left(d\left(v, y_{o}\right) / t\right)$ and observe that, thanks to the previous observation, $d\left(v, y_{0}\right) \leq r$. It follows that (choosing $y=y_{o}$ ),

$$
\begin{aligned}
Q_{t} f(u)-Q_{t} f(v) & =\inf _{y}\{f(y)+t \alpha(d(u, y) / t)\}-f\left(y_{o}\right)-t \alpha\left(d\left(v, y_{o}\right) / t\right) \\
& \leq t \alpha\left(d\left(u, y_{o}\right) / t\right)-t \alpha\left(d\left(v, y_{o}\right) / t\right) \leq \alpha^{\prime}(2 r / t) d(u, v),
\end{aligned}
$$

which proves that $\operatorname{Lip}\left(Q_{t} f, r\right)<\infty$.

Now assume that $\alpha(h) / h \rightarrow \ell \in \mathbb{R}^{+}$, when $h \rightarrow \infty$ and let us prove that $Q_{t} f$ is $\ell$-Lipschitz. The convexity of $\alpha$ implies that

$$
\frac{\alpha(h)}{h} \leq \alpha^{\prime}(h) \leq \frac{\alpha(2 h)-\alpha(h)}{h}, \quad \forall h>0 .
$$

So $\sup _{h} \alpha^{\prime}(h)=\lim _{h \rightarrow \infty} \alpha^{\prime}(h)=\ell$ and it follows that $Q_{t} f$ is $\ell$-Lipschitz as an infimum of $\ell$-Lipschitz functions.

(1) The inequality (A.5) above also proves that $r_{\alpha}=1$ when $\ell<+\infty$.

(3) Let $\left(\lambda_{n}\right)_{n \geq 0}$ be a sequence of real numbers converging to 1 . For any $x \in X$, let $m(t, x)$ be the set of points $y \in X$ such that

$$
Q_{t} f(x)=\inf _{z \in X}\{f(z)+t \alpha(d(x, z) / t)\}=f(y)+t \alpha(d(x, y) / t) .
$$


For any $n$, let $y_{n}$ be such that $Q_{t}\left(\lambda_{n} f\right)(x)=\lambda_{n} f\left(y_{n}\right)+t \alpha\left(d\left(x, y_{n}\right) / t\right)$. We have, for all $z \in X$,

$$
\lambda_{n} \inf f+t \alpha\left(d\left(x, y_{n}\right) / t\right) \leq \lambda_{n} f\left(y_{n}\right)+t \alpha\left(d\left(x, y_{n}\right) / t\right) \leq \lambda_{n} f(z)+t \alpha(d(x, z) / t) .
$$

Since $\left(\lambda_{n}\right)_{n}$ converges, we deduce that the sequence $\left(y_{n}\right)_{n}$ is bounded. Let $y$ be a limit point of a converging subsequence of $\left(y_{n}\right)_{n}$. Passing to the limit leads to

$$
f(y)+t \alpha(d(x, y) / t) \leq f(z)+t \alpha(d(x, z) / t) \quad \forall z \in X .
$$

Hence, $y \in m(t, x)$. In turn, after easy considerations left to the reader, one sees that $Q_{t}\left(\lambda_{n} f\right)(x) \rightarrow Q_{t} f(x)$, when $n \rightarrow \infty$ as expected. The conclusion of point (2) follows and the proof is complete.

\section{References}

[1] Ambrosio, L., Gigli, N. and Savaré, G.: Calculus and heat flow in metric measure spaces and applications to spaces with Ricci bounds from below. Invent. Math. 195 (2014), no. 2, 289-391.

[2] Ambrosio, L., Gigli, N. And Savaré, G.: Density of Lipschitz functions and equivalence of weak gradients in metric measure spaces. Rev. Mat. Iberoam. 29 (2013), no. 3, 969-996.

[3] Balogh, Z., Engoulatov, A., Hunziker, L., and Maasalo, O. E.: Functional inequalities and Hamilton-Jacobi equations in geodesic spaces. Potential Anal. 36 (2012), no. 2, 317-337.

[4] Bobkov, S. G., Gentil, I. And Ledoux, M.: Hypercontractivity of HamiltonJacobi equations. J. Math. Pures Appl. (9) 80 (2001), no. 7, 669-696.

[5] Bobkov, S. Gं. AND Götze, F.: Exponential integrability and transportation cost related to logarithmic Sobolev inequalities. J. Funct. Anal. 163 (1999) no. 1, 1-28.

[6] Djellout, H., Guillin, A., And Wu, L.: Transportation cost-information inequalities and applications to random dynamical systems and diffusions. Ann. Probab. 32 (2004), no. 3B, 2702-2732.

[7] Evans, L. C.: Partial differential equations.. Graduate Studies in Mathematics, 19. American Mathematical Society, Providence, RI, 1998.

[8] Gentil, I., Guillin, A., And Miclo, L.: Modified logarithmic Sobolev inequalities and transportation inequalities. Probab. Theory Related Fields 133 (2005), no. 3, 409-436.

[9] Gigli, N. And Ledoux, M.: From log Sobolev to Talagrand: a quick proof. Discrete Contin. Dyn. Syst. 33 (2013), no. 5, 1927-1935.

[10] Gozlan, N.: A characterization of dimension free concentration in terms of transportation inequalities. Ann. Probab. 37 (2009), no. 6, 2480-2498.

[11] Gozlan, N., Roberto, C. And Samson, P. M.: A new characterization of Talagrand's transport-entropy inequalities and applications. Ann. Probab. 39 (2011), no. $3,857-880$.

[12] Gozlan, N., Roberto, C., and Samson, P. M.: Characterization of Talagrand's transport-entropy inequalities in metric spaces. Ann. Probab. 41 (2013), no. 5, $3112-3139$ 
[13] Gross, L.: Logarithmic Sobolev inequalities. Amer. J. Math. 97 (1975), no. 4, 1061-1083.

[14] Lott, J. And Villani, C.: Hamilton-Jacobi semigroup on length spaces and applications. J. Math. Pures Appl. (9) 88 (2007), no. 3, 219-229.

[15] Marton, K.: A simple proof of the blowing-up lemma. IEEE Trans. Inform. Theory 32 (1986), no. 3, 445-446.

[16] Отto, F. And Villani, C.: Generalization of an inequality by Talagrand and links with the logarithmic Sobolev inequality. J. Funct. Anal. 173 (2000), no. 2, 361-400.

[17] RaO, M. M. And Ren, Z. D.: Theory of Orlicz spaces. Monographs and Textbooks in Pure and Applied Mathematics 146, Marcel Dekker, New York, 1991.

[18] Rockafellar, T.R.: Convex analysis. Princeton Landmarks in Mathematics, Princeton University Press, Princeton, NJ, 1997. Reprint of the 1970 original, Princeton Paperbacks.

[19] Talagrand, M.: Transportation cost for Gaussian and other product measures. Geom. Funct. Anal. 6 (1996), no. 3, 587-600.

[20] Villani, C.: Optimal transport. Old and New. Grundlehren der Mathematischen Wissenschaften 338, Springer-Verlag, Berlin, 2009.

[21] Wang, F. Y.: Probability distance inequalities on Riemannian manifolds and path spaces. J. Funct. Anal. 206 (2004), no. 1, 167-190.

Received March 21, 2012; revised September 2, 2012.

Nathael Gozlan: Université Paris-Est, LAMA (UMR 8050), UPEMLV, UPEC, CNRS, F-77454, Marne-la-Vallée, France.

E-mail: nathael.gozlan@univ-mlv.fr

Cyril Roberto: Université Paris Ouest Nanterre la Défense, MODAL'X, EA 3454, 200 avenue de la République, 92000 Nanterre, France.

E-mail: croberto@math.cnrs.fr

PAul-Marie SAmson: Université Paris-Est, LAMA (UMR 8050), UPEMLV, UPEC, CNRS, F-77454, Marne-la-Vallée, France.

E-mail: paul-marie.samson@univ-mlv.fr

The authors were partially supported by the "Agence Nationale de la Recherche" through the grants ANR 2011 BS01 00701 and ANR 10 LABX-58; the second author was partially supported by the European Research Council through the "Advanced Grant" PTRELSS 228032. 